\title{
The HDUV Survey: A Revised Assessment of the Relationship between UV Slope and Dust Attenuation for High-redshift Galaxies
}

\author{
Naveen A. Reddy ${ }^{1,10}$ (), Pascal A. Oesch ${ }^{2,3}$ (), Rychard J. Bouwens ${ }^{4}$ (D), Mireia Montes ${ }^{3}$, Garth D. Illingworth ${ }^{5}$, \\ Charles C. Steidel ${ }^{6}$ (i) , Pieter G. van Dokkum ${ }^{3}$ (i) , Hakim Atek ${ }^{3}$ (i), Marcella C. Carollo ${ }^{7}$ (i), Anna Cibinel ${ }^{8}$ (i), \\ Brad Holden $^{5}\left(\mathbb{D}\right.$, Ivo Labbé ${ }^{4}\left(\mathbb{D}\right.$, Dan Magee ${ }^{5}$, Laura Morselli ${ }^{9}$, Erica J. Nelson ${ }^{9}$ (1), and Steve Wilkins ${ }^{8}$ \\ ${ }^{1}$ Department of Physics and Astronomy, University of California, Riverside, 900 University Avenue, Riverside, CA 92521, USA; naveenr@ucr.edu \\ ${ }^{2}$ Geneva Observatory, Université de Genève, Chemin des Maillettes 51, 1290 Versoix, Switzerland \\ ${ }^{3}$ Yale Center for Astronomy and Astrophysics, Yale University, New Haven, CT 06511, USA \\ ${ }^{4}$ Leiden Observatory, Leiden University, NL-2300 RA Leiden, The Netherlands \\ ${ }^{5}$ UCO/Lick Observatory, University of California, Santa Cruz, 1156 High St., Santa Cruz, CA 95064, USA \\ ${ }^{6}$ Cahill Center for Astronomy and Astrophysics, California Institute of Technology, MS 249-17, Pasadena, CA 91125, USA \\ ${ }^{7}$ Institute for Astronomy, ETH Zurich, 8092 Zurich, Switzerland \\ ${ }^{8}$ Astronomy Centre, Department of Physics and Astronomy, University of Sussex, Brighton, BN1 9QH, UK \\ ${ }^{9}$ Max Planck Institute for Extraterrestrial Physics, Giessenbachstrasse, D-85741 Garching bei München, Germany \\ Received 2017 May 24; revised 2017 November 28; accepted 2017 December 18; published 2018 January 23
}

\begin{abstract}
We use a newly assembled sample of 3545 star-forming galaxies with secure spectroscopic, grism, and photometric redshifts at $z=1.5-2.5$ to constrain the relationship between UV slope $(\beta)$ and dust attenuation $\left(L_{\mathrm{IR}} / L_{\mathrm{UV}} \equiv \mathrm{IRX}\right)$. Our sample significantly extends the range of $L_{\mathrm{UV}}$ and $\beta$ probed in previous UV-selected samples, including those as faint as $M_{1600}=-17.4\left(\simeq 0.05 L_{\mathrm{UV}}^{*}\right)$ and $-2.6 \lesssim \beta \lesssim 0.0$. IRX is measured using stacks of deep Herschel data, and the results are compared with predictions of the IRX $-\beta$ relation for different assumptions of the stellar population model and obscuration curve. We find that $z=1.5-2.5$ galaxies have an IRX $-\beta$ relation that is consistent with the predictions for an SMC curve if we invoke subsolar-metallicity models currently favored for high-redshift galaxies, while the commonly assumed starburst curve overpredicts the IRX at a given $\beta$ by a factor of $\gtrsim 3$. IRX is roughly constant with $L_{\mathrm{UV}}$ for $L_{\mathrm{UV}} \gtrsim 3 \times 10^{9} L_{\odot}$. Thus, the commonly observed trend of fainter galaxies having bluer $\beta$ may simply reflect bluer intrinsic slopes for such galaxies, rather than lower obscurations. The IRX $-\beta$ relation for young/low-mass galaxies at $z \gtrsim 2$ implies a dust curve that is steeper than the SMC. The lower attenuations and higher ionizing photon output for low-metallicity stellar populations point to Lyman continuum production efficiencies, $\xi_{\text {ion }}$, that may be elevated by a factor of $\approx 2$ relative to the canonical value for $L^{*}$ galaxies, aiding in their ability to keep the universe ionized at $z \sim 2$.
\end{abstract}

Key words: dark ages, reionization, first stars - dust, extinction - galaxies: evolution - galaxies: formation galaxies: high-redshift - galaxies: ISM

\section{Introduction}

The ultraviolet (UV) spectral slope, $\beta$, where $f_{\lambda} \propto \lambda^{\beta}$, is by far the most commonly used indicator of dust obscurationusually parameterized as the ratio of the infrared to UV luminosity, $L_{\mathrm{IR}} / L_{\mathrm{UV}}$, or "IRX" (Calzetti et al. 1994; Meurer et al. 1999) — in moderately reddened high-redshift $(z \gtrsim 1.5)$ star-forming galaxies. The UV slope can be measured easily from the same photometry used to select galaxies based on the Lyman break, and the slope can be used as a proxy for the dust obscuration in galaxies (e.g., Calzetti et al. 1994; Meurer et al. 1999; Adelberger \& Steidel 2000; Reddy et al. 2006b, 2010, 2012a; Daddi et al. 2007; Overzier et al. 2011; Buat et al. 2012) whose dust emission is otherwise too faint to directly detect in the mid- and far-infrared (e.g., Adelberger \& Steidel 2000; Reddy et al. 2006b). Generally, these studies have indicated that UV-selected star-forming galaxies at redshifts $1.5 \lesssim z \lesssim 3.0$ follow on average the relationship between UV slope and dust obscuration (i.e., the IRX- $\beta$ relation) found for local UV starburst galaxies (e.g., Nandra et al. 2002; Reddy \& Steidel 2004; Reddy et al. 2006b; Daddi et al. 2007; Sklias et al. 2014; see also Heinis et al. 2013; Álvarez-Márquez et al. 2016), though with some deviations

${ }^{10}$ Alfred P. Sloan Research Fellow. that depend on galaxy age (Reddy et al. 2006b, 2010; Siana et al. 2008; Buat et al. 2012), bolometric luminosity (e.g., Chapman et al. 2005; Reddy et al. 2006b; Casey et al. 2014b), stellar mass (Pannella et al. 2009; Reddy et al. 2010; Bouwens et al. 2016a), and redshift (Pannella et al. 2015). Unfortunately, typical star-forming $\left(L^{*}\right)$ galaxies at these redshifts are too faint to directly be detected in the far-infrared. As such, with the exception of individual lensed galaxy studies (Siana et al. 2008, 2009; Sklias et al. 2014; Watson et al. 2015; DessaugesZavadsky et al. 2017), most investigations that have explored the relation between UV slope and dust obscuration for moderately reddened galaxies have relied on stacking relatively small numbers of objects and/or used shorter-wavelength emission-such as that arising from polycyclic aromatic hydrocarbons (PAHs) — to infer infrared luminosities.

New avenues of exploring the dustiness of high-redshift galaxies have been made possible with facilities such as the Atacama Large Millimeter Array (ALMA), allowing for direct measurements of either the dust continuum or far-IR spectral features for more typical star-forming galaxies in the distant universe (Carilli \& Walter 2013; Bouwens et al. 2016a; Dunlop et al. 2017; Fudamoto et al. 2017). Additionally, the advent of large-scale rest-optical spectroscopic surveys of intermediateredshift galaxies at $1.4 \lesssim z \lesssim 2.6$ - such as the 3D-HST (van Dokkum et al. 2013), the MOSFIRE Deep Evolution Field 
(MOSDEF; Kriek et al. 2015), and the Keck Baryonic Structure surveys (Steidel et al. 2014) — has enabled measurements of obscuration in individual high-redshift star-forming galaxies using Balmer recombination lines (e.g., Price et al. 2014; Reddy et al. 2015; Nelson et al. 2016). While these nebular line measurements will be possible in the near future for $z \gtrsim 3$ galaxies with the James Webb Space Telescope (JWST), the limited lifetime of this facility and the targeted nature of both ALMA far-IR and JWST near- and mid-IR observations mean that the UV slope will remain the only readily accessible direct measurement of reddening for large numbers of individual typical galaxies at $z \gtrsim 3$ in the foreseeable future.

Despite the widespread use of the UV slope to infer dust attenuation, there are several complications associated with its use. First, the UV slope is sensitive to metallicity and star formation history (e.g., Kong et al. 2004; Seibert et al. 2005; Johnson et al. 2007; Dale et al. 2009; Muñoz-Mateos et al. 2009; Reddy et al. 2010, 2012b; Wilkins et al. 2011, 2013; Boquien et al. 2012; Reddy et al. 2012b; Grasha et al. 2013; Schaerer et al. 2013; Zeimann et al. 2015). Second, there is evidence that the relationship between UV slope and dust obscuration depends on stellar mass and/or age (e.g., Reddy et al. 2006b; Buat et al. 2012; Zeimann et al. 2015; Bouwens et al. 2016a), perhaps reflecting variations in the shape of the attenuation curve. Third, the measurement of the UV slope may be complicated by the presence of the $2175 \AA$ absorption feature (Noll et al. 2009; Buat et al. 2011, 2012; Kriek \& Conroy 2013; Reddy et al. 2015). Fourth, as noted above, independent inferences of the dust attenuation in faint galaxies typically involve stacking mid- and far-IR data, but such stacking masks the scatter in the relationship between UV slope and obscuration. Quantifying this scatter can elucidate the degree to which the attenuation curve may vary from galaxy to galaxy, or highlight the sensitivity of the UV slope to factors other than dust obscuration. In general, the effects of age, metallicity, and star formation history on the UV slope may become important for ultrafaint galaxies at high redshift, which have been suggested to undergo bursty star formation (e.g., Weisz et al. 2012; Hopkins et al. 2014; Domínguez et al. 2015; Guo et al. 2016; Sparre et al. 2017; Faucher-Giguere 2018).

Obtaining direct constraints on the dust obscuration of UVfaint galaxies is an important step in evaluating the viability of the UV slope to trace dustiness, quantifying the bolometric luminosities of ultrafaint galaxies and their contribution to the global star formation rate (SFR) and stellar mass densities, assessing possible variations in the dust obscuration curve over a larger dynamic range of galaxy characteristics (e.g., SFR, stellar mass, age, metallicity), and discerning the degree to which the UV slope may be affected by short-timescale variations in SFR.

Separately, recent advances in stellar population models that include realistic treatments of stellar mass loss, rotation, and multiplicity (Eldridge \& Stanway 2009; Brott et al. 2011; Levesque et al. 2012; Leitherer et al. 2014) can result in additional dust heating from ionizing and/or recombination photons. Moreover, the intrinsic UV spectral slopes of highredshift galaxies with lower stellar metallicities may be substantially bluer (Schaerer et al. 2013; Alavi et al. 2014; de Barros et al. 2014; Sklias et al. 2014; Cullen et al. 2017) than what has been typically assumed in studies of the IRX $-\beta$ relation. Thus, it seems timely to re-evaluate the $\mathrm{IRX}-\beta$ relation in light of these issues.

With this in mind, we use a newly assembled large sample of galaxies with secure spectroscopic or photometric redshifts at $1.5 \leqslant z \leqslant 2.5$ in the GOODS-North and GOODS-South fields to investigate the correlation between UV slope and dust obscuration. Our sample takes advantage of newly acquired Hubble UVIS F275W and F336W imaging from the HDUV survey (P. A. Oesch et al. 2018, in preparation), which aids in determining photometric redshifts when combined with existing 3D-HST photometric data. This large sample enables precise measurements of dust obscuration through the stacking of far-infrared images from the Herschel Space Observatory and also enables stacking in multiple bins of other galaxy properties (e.g., stellar mass, UV luminosity) to investigate the scatter in the IRX $-\beta$ relation. We also consider the newest stellar population models-those that may be more appropriate in describing very high redshift $(z \gtrsim 2)$ galaxies - in interpreting the relationship between UV slope and obscuration.

The outline of this paper is as follows. In Section 2, we discuss the selection and modeling of stellar populations of galaxies used in this study. The methodology used for stacking the mid- and far-IR Spitzer and Herschel data is discussed in Section 3. In Section 4, we calculate the predicted relationships between IRX and $\beta$ for different attenuation/extinction curves using energy balance arguments. These predictions are compared to our (as well as literature) stacked measurements of IRX in Section 5. In this section, we also consider the variation of IRX with stellar masses, UV luminosities, and the ages of galaxies, as well as the implications of our results for modeling the stellar populations and inferring the ionizing efficiencies of highredshift galaxies. AB magnitudes are assumed throughout (Oke \& Gunn 1983), and we use a Chabrier (2003) initial mass function (IMF) unless stated otherwise. We adopt a cosmology with $H_{0}=70 \mathrm{~km} \mathrm{~s}^{-1} \mathrm{Mpc}^{-1}, \Omega_{\Lambda}=0.7$, and $\Omega_{\mathrm{m}}=0.3$.

\section{Sample and IR Imaging}

\subsection{Parent Sample}

A few basic properties of our sample are summarized in Table 1. Our sample of galaxies was constructed by combined the publicly available ground- and space-based photometry compiled by the 3D-HST survey (Skelton et al. 2014) with newly obtained imaging from the Hubble Deep UV (HDUV) Legacy Survey (GO-13871; P. A. Oesch et al. 2018, in preparation). The HDUV survey imaged the two GOODS fields in the F275W and F336W bands to depths of $\simeq 27.5$ and $27.9 \mathrm{mag}$, respectively $(5 \sigma ; 0$ ". 4 diameter aperture), with the UVIS channel of the Hubble Space Telescope (HST) WFC3 instrument. A significant benefit of the HDUV imaging is that it allows for the Lyman break selection of galaxies to fainter UV luminosities and lower redshifts than possible from ground-based surveys (P. A. Oesch et al. 2018, in preparation) and builds on previous efforts to use deep UVIS imaging to select Lyman break galaxies (LBGs) at $z \sim 2$ (Hathi et al. 2010; Windhorst et al. 2011). The reduced UVIS images, covering $\approx 100 \mathrm{arcmin}^{2}$, include previous imaging obtained by the CANDELS (Koekemoer et al. 2011) and UVUDF surveys (Teplitz et al. 2013; Rafelski et al. 2015). 
Table 1

Sample Characteristics

\begin{tabular}{|c|c|}
\hline Property & Value \\
\hline Fields & GOODS-N, GOODS-S \\
\hline Total area & $\sim 329 \operatorname{arcmin}^{2}$ \\
\hline Area with HDUV imaging & $\sim 100 \operatorname{arcmin}^{2}$ \\
\hline UV/optical photometry & $\begin{array}{l}\text { 3D- } H S T \text { Catalogs }{ }^{\mathrm{a}} \text { and } \mathrm{HDUV}^{\mathrm{b}} \mathrm{F} 275 \mathrm{~W} \\
\text { and F336W }\end{array}$ \\
\hline Mid-IR imaging & Spitzer GOODS Imaging Program ${ }^{c}$ \\
\hline Far-IR imaging & GOODS-Herschel $^{\mathrm{d}}$ and PEP ${ }^{\mathrm{e}}$ Surveys \\
\hline Optical depth of sample & $H \simeq 27$ \\
\hline UV depth of sample & $m_{\mathrm{UV}} \simeq 27$ \\
\hline Total number of galaxies & 4078 \\
\hline $\begin{array}{l}\text { Number of galaxies with far-IR } \\
\text { coverage }\end{array}$ & 3569 \\
\hline $\begin{array}{l}\text { Final number (excl. far-IR- } \\
\text { detected objects) }\end{array}$ & 3545 \\
\hline$\beta$ Range & $-2.55 \leqslant \beta \leqslant 1.05(\langle\beta\rangle=-1.71)$ \\
\hline
\end{tabular}

Notes.

a Skelton et al. (2014).

${ }^{b}$ P. A. Oesch et al. (2018, in preparation).

${ }^{c}$ PI: Dickinson.

d Elbaz et al. (2011).

e PI: Lutz, Magnelli et al. (2013).

\subsection{Photometry and Stellar Population Parameters}

Source Extractor (Bertin \& Arnouts 1996) was used to measure photometry on the UVIS images using the detection maps for the combined $\mathrm{F} 125 \mathrm{~W}+\mathrm{F} 140 \mathrm{~W}+\mathrm{F} 160 \mathrm{~W}$ images, as was done for the 3D-HST photometric catalogs (Skelton et al. 2014). The publicly available 3D-HST photometric catalogs were then updated with the HDUV photometry-i.e., such that the updated catalogs contain updated photometry for objects lying in the HDUV pointings, as well as the original set of photometry for objects lying outside the HDUV pointings. This combined data set was then used to calculate photometric redshifts using EAZY (Brammer et al. 2008) and determine stellar population parameters (e.g., stellar mass) using FAST (Kriek et al. 2009). Where available, grism and external spectroscopic redshifts were used in lieu of the photometric redshifts when fitting for the stellar populations. These external spectroscopic redshifts are provided in the 3D-HST catalogs (Momcheva et al. 2016). We also included 759 spectroscopic redshifts for galaxies observed during the 2012B-2015A semesters of the MOSDEF survey (Kriek et al. 2015).

For the stellar population modeling, we adopted the Conroy \& Gunn (2010) stellar population models for $Z=0.019 \mathrm{Z}_{\odot}$, a delayed- $\tau$ star formation history with $8.0 \leqslant \log [\tau /$ year $] \leqslant$ 10.0, a Chabrier (2003) IMF, and the Calzetti et al. (2000) dust attenuation curve with $0.0 \leqslant A_{\mathrm{V}} \leqslant 4.0 .^{11}$ We imposed a minimum age of $40 \mathrm{Myr}$ based on the typical dynamical timescale for $z \sim 2$ galaxies (Reddy et al. 2012b).

The UV slope for each galaxy was calculated both by (a) fitting a power law through the broadband photometry, including only bands lying redward of the Lyman break and blueward of rest-frame $2600 \mu \mathrm{m}$, and (b) fitting a power law through the best-fit spectral energy distribution (SED) points

\footnotetext{
11 Below, we consider the effect of stellar population age on the IRX $-\beta$ relations. In that context, the ages derived for the vast majority of galaxies in our sample are within $\delta \log$ [Age/year] $\simeq 0.1$ dex of those derived assuming a constant star formation history.
}

that lie in wavelength windows spanning rest-frame 1268 $\AA \leqslant \lambda \leqslant 2580 \AA$, as defined in Calzetti et al. (1994). Method (a) includes a more conservative estimate for the errors in $\beta$, but generally the two methods yielded values of the UV slope for a given galaxy that were within $\delta \beta \simeq 0.1$ of each other. We adopted the $\beta$ calculated using method (a) for the remainder of our analysis, and note that in Section 4 we consider the value of $\beta$ using windows lying strictly blueward of $\approx 1800 \AA$. The median uncertainty in the photometrically derived $\beta$ is $\sigma_{\beta} \simeq 0.19$.

\subsection{Criteria for Final Sample}

The photometric catalogs, along with those containing the redshifts and stellar population parameters, were used to select galaxies based on the following criteria. First, the object must have a Source Extractor "class star" parameter $<0.95$, or observed-frame $U-J$ and $J-K$ colors that reside in the region occupied by star-forming galaxies as defined by Skelton et al. (2014) — these criteria ensure the removal of stars.

Second, the galaxy must have a spectroscopic or grism redshift, or $95 \%$ confidence intervals in the photometric redshift, that lie in the range $1.5 \leqslant z \leqslant 2.5$. Note that the high photometric redshift confidence intervals required for inclusion in our sample naturally selects those objects with $H \lesssim 27$.

Third, the object must not have a match in X-ray active galactic nucleus (AGN) catalogs compiled for the GOODSNorth and GOODS-South fields (e.g., Shao et al. 2010; Xue et al. 2011). Additionally, we use the Donley et al. (2012) Spitzer IRAC selection to isolate any infrared-bright AGNs. While the X-ray and IRAC selections may not identify all AGNs at the redshifts of interest, they are likely to isolate those AGNs that may significantly influence our stacked far-IR measurements.

Fourth, the object must not have rest-frame $U-V$ and $V-J$ colors that classify it as a quiescent galaxy (Williams et al. 2009; Skelton et al. 2014). The object is further required to have a specific SFR (sSFR) $\gtrsim 0.1 \mathrm{Gyr}^{-1}$. These criteria safeguard against the inclusion of galaxies where $\beta$ may be red owing to the contribution of older stars to the near-UV continuum, or where dust heating by older stars may become significant.

Fifth, to ensure that the sample is not biased toward objects with red $U-H$ colors at faint $U$ magnitudes (owing to the limit in $H$-band magnitude mentioned previously), the galaxy must have an apparent magnitude at $[1+z] \times 1600 \AA$ of $\leqslant 27.0 \mathrm{mag}$. This limit still allows us to include galaxies with absolute magnitudes as faint as $M_{1600} \simeq-17.4$. These criteria result in a sample of 4078 galaxies.

\subsection{Spitzer and Herschel Imaging}

We used the publicly available Spitzer/MIPS $24 \mu \mathrm{m}$ and Herschel/PACS 100 and $160 \mu \mathrm{m}$ data in the two GOODS fields for our analysis. The $24 \mu \mathrm{m}$ data come from the Spitzer GOODS imaging program (PI: Dickinson) and trace the dustsensitive rest-frame $7.7 \mu \mathrm{m}$ emission feature for galaxies at $1.5 \leqslant z \leqslant 2.5$ (e.g., Reddy et al. 2006b). The observed $24 \mu \mathrm{m}$ fluxes of $z \sim 2$ galaxies have been used extensively in the past to derive infrared luminosities $\left(L_{\mathrm{IR}}\right)$ given the superior sensitivity of these data to dust emission when compared with observations taken at longer wavelengths (roughly a factor of three times more sensitive than Herschel/PACS to galaxies of 
a given $L_{\mathrm{IR}}$ at $z \sim 2$; Elbaz et al. 2011). However, a number of observations have highlighted the strong variation in $L_{7.7} / L_{\mathrm{IR}}$ with SFR (Rieke et al. 2009; Shipley et al. 2016), SFR surface density (Elbaz et al. 2011), and gas-phase metallicity and ionization parameter at high redshift (Shivaei et al. 2017). As such, while we stacked the $24 \mu \mathrm{m}$ data for galaxies in our sample, we did not consider these measurements when calculating $L_{\mathrm{IR}}$. In Appendix $\mathrm{B}$, we consider further the variation in $L_{7.7} / L_{\mathrm{IR}}$ with other galaxy characteristics.

The Herschel data come from the GOODS-Herschel Open Time Key Program (Elbaz et al. 2011) and the PACS Evolutionary Probe (PEP) Survey (PI: Lutz; Magnelli et al. 2013) and probe the rest-frame $\simeq 30-65 \mu \mathrm{m}$ dust continuum emission for galaxies at $1.5 \leqslant z \leqslant 2.5$. We chose not to use the SPIRE data in our analysis given the much coarser spatial resolution of these data $\left(\mathrm{FWHM} \gtrsim 18^{\prime \prime}\right)$ relative to the $24 \mu \mathrm{m}(\mathrm{FWHM} \simeq 5$ ". 4), $100 \mu \mathrm{m}$ (FWHM $\simeq 6$ ". 7), and $160 \mu \mathrm{m}\left(\mathrm{FWHM} \simeq 11^{\prime \prime}\right)$ data (see Heinis et al. 2014; ÁlvarezMárquez et al. 2016). In particular, the bias factors (see Section 3) derived for the SPIRE stacks of our galaxies were typically larger than 50\%. Furthermore, we found that the systematic differences in the stacked fluxes obtained from SPIRE residual images that were constructed using Spitzer/ MIPS $24 \mu \mathrm{m}$ and Herschel/PACS 100/160 $\mu \mathrm{m}$ were larger than the stacked fluxes themselves. Having said this, future observations (e.g., with ALMA) that constrain the longerwavelength (i.e., Rayleigh-Jeans tail) dust emission will be beneficial for obtaining more precise estimates of $L_{\mathrm{IR}}$ when combined with existing Herschel data. For the Herschel data used in our analysis, the pixel scales of the 24, 100, and $160 \mu \mathrm{m}$ images are $1 . \prime 2,1$ "' 2 , and 2 ". 4 , respectively. As noted above, only the 100 and $160 \mu \mathrm{m}$ data are used to calculate $L_{\mathrm{IR}}$.

Of the 4078 galaxies in the sample discussed above, 3569 lie within the portions of the Herschel imaging that are $80 \%$ complete to flux levels of 1.7 and $5.5 \mathrm{mJy}$ for the 100 and $160 \mu \mathrm{m}$ maps in GOODS-N, respectively, and 1.3 and $3.9 \mathrm{mJy}$ for the 100 and $160 \mu \mathrm{m}$ maps in GOODS-S, respectively. Of these galaxies, 24 (or $0.67 \%$ ) are directly detected with signalto-noise ratio $(\mathrm{S} / \mathrm{N})>3$ in either the 100 or $160 \mu \mathrm{m}$ images. As we are primarily concerned with constraining the IRX $-\beta$ relation for moderately reddened galaxies, we removed all directly detected Herschel objects from our sample-the latter are very dusty star-forming galaxies at the redshifts of interest with $L_{\mathrm{IR}} \gtrsim 10^{12} L_{\odot}$. The very low frequency of infraredluminous objects among UV-faint galaxies in general could have been anticipated from the implied low number density of $L_{\mathrm{IR}} \gtrsim 10^{12} L_{\odot}$ objects from the IR luminosity function (Reddy et al. 2008; Magnelli et al. 2013) and the high number density of UV-faint galaxies inferred from the UV luminosity function (Reddy et al. 2008; Reddy \& Steidel 2009; Alavi et al. 2016) at $z \sim 2$. The inclusion of such dusty galaxies does not significantly affect our stacking analysis owing to the very small number of such objects. More generally speaking, at a given faint UV luminosity, galaxies with intrinsically low bolometric luminosities will vastly outnumber those with intrinsically high bolometric luminosities owing to the shapes of the IR and UV luminosity functions (e.g., Reddy et al. 2008). Thus, the relations between dustiness and UV slope presented in our subsequent analysis should remain valid for samples that are dominated by UV-faint galaxies (like the one analyzed here). Excluding these dusty galaxies, our final sample consists of 3545 galaxies, with the redshift and absolute magnitude distributions shown in Figure 1.

\subsection{Summary of Sample}

To summarize, we have combined HDUV UVIS and 3DHST cataloged photometry to constrain photometric redshifts for galaxies in the GOODS fields and isolate those star-forming galaxies with redshifts $z=1.5-2.5$ down to a limiting near-IR magnitude of $\simeq 27 \mathrm{AB}$ (Table 1 ). All galaxies are significantly detected (with $\mathrm{S} / \mathrm{N}>3$ ) down to an observed optical (restframe UV) magnitude of $27 \mathrm{AB}$. Our sample includes objects with spectroscopic redshifts in the aforementioned range wherever possible. This sample is then used as a basis for stacking deep Herschel data, as discussed in the next section.

One of the most beneficial attributes of our sample is that it contains the largest number of UV-faint galaxies-extending up to $\approx 3$ mag fainter than the characteristic absolute magnitude at $z \sim 2.3\left(M_{1700}^{*}=-20.70\right.$; Reddy \& Steidel 2009) and $z \sim 1.9\left(M_{1500}^{*}=-20.16\right.$; Oesch et al. 2010)—with robust redshifts at $1.5 \leqslant z \leqslant 2.5$ assembled to date (Figure 1 ). The general faintness of galaxies in our sample is underscored by their very low detection rate $(\mathrm{S} / \mathrm{N}>3)$ at $24 \mu \mathrm{m}-85$ of 3545 galaxies, or $\approx 2.4 \%$ - compared to the $\approx 40 \%$ detection rate for rest-frame UV-selected galaxies with $\mathcal{R} \leqslant 25.5$ (Reddy et al. 2010). Consequently, unlike most previous efforts using ground-based UV-selected samples of limited depth, the HDUV sample presents a unique opportunity to evaluate the IRX $-\beta$ relation for the analogs of the very faint galaxies that dominate the UV and bolometric luminosity densities at $z \gg 3$ (e.g., Reddy et al. 2008; Smit et al. 2012), but for which direct constraints on their infrared luminosities are either difficult to obtain or subject to biases (e.g., dust temperature; Capak et al. 2015; Bouwens et al. 2016a; Ferrara et al. 2016) that are more severe than at $z \sim 2$.

\section{Stacking Methodology}

To mitigate any systematics in the stacked fluxes due to bright objects proximate to the galaxies in our sample, we performed the stacking on residual images that were constructed as follows. ${ }^{12}$ We used the $24 \mu \mathrm{m}$ catalogs and pointspread functions (PSFs) included in the GOODS-Herschel data release to subtract all objects with $\mathrm{S} / \mathrm{N}>3$ in the $24 \mu \mathrm{m}$ images, with the exception of the 85 objects in our sample that are directly detected at $24 \mu \mathrm{m}$. Objects with $\mathrm{S} / \mathrm{N}>3$ in the $24 \mu \mathrm{m}$ images were used as priors to fit and subtract objects with $\mathrm{S} / \mathrm{N}>3$ in the 100 and $160 \mu \mathrm{m}$ images. The result is a set of residual images at 24, 100, and $160 \mu \mathrm{m}$ for both GOODS fields.

For each galaxy contributing to the stack, we extracted from the 24,100 , and $160 \mu \mathrm{m}$ residual images regions of $41 \times 41$, $52 \times 52$, and $52 \times 52$ pixels, respectively, centered on the galaxy. The subimages were then divided by the UV luminosity, $L_{\mathrm{UV}}=\nu L_{\nu}$ at $1600 \AA$, of the galaxy, and these normalized subimages for each band were then averaged together using $3 \sigma$ clipping for all the objects in the stack. We performed PSF photometry on the stacked images to measure the fluxes. Because the images are normalized by $L_{\mathrm{UV}}$, the stacked fluxes are directly proportional to the average

\footnotetext{
12 As discussed in Reddy et al. (2012a), stacking on the science images themselves yields results similar to those obtained by stacking on the residual images.
} 

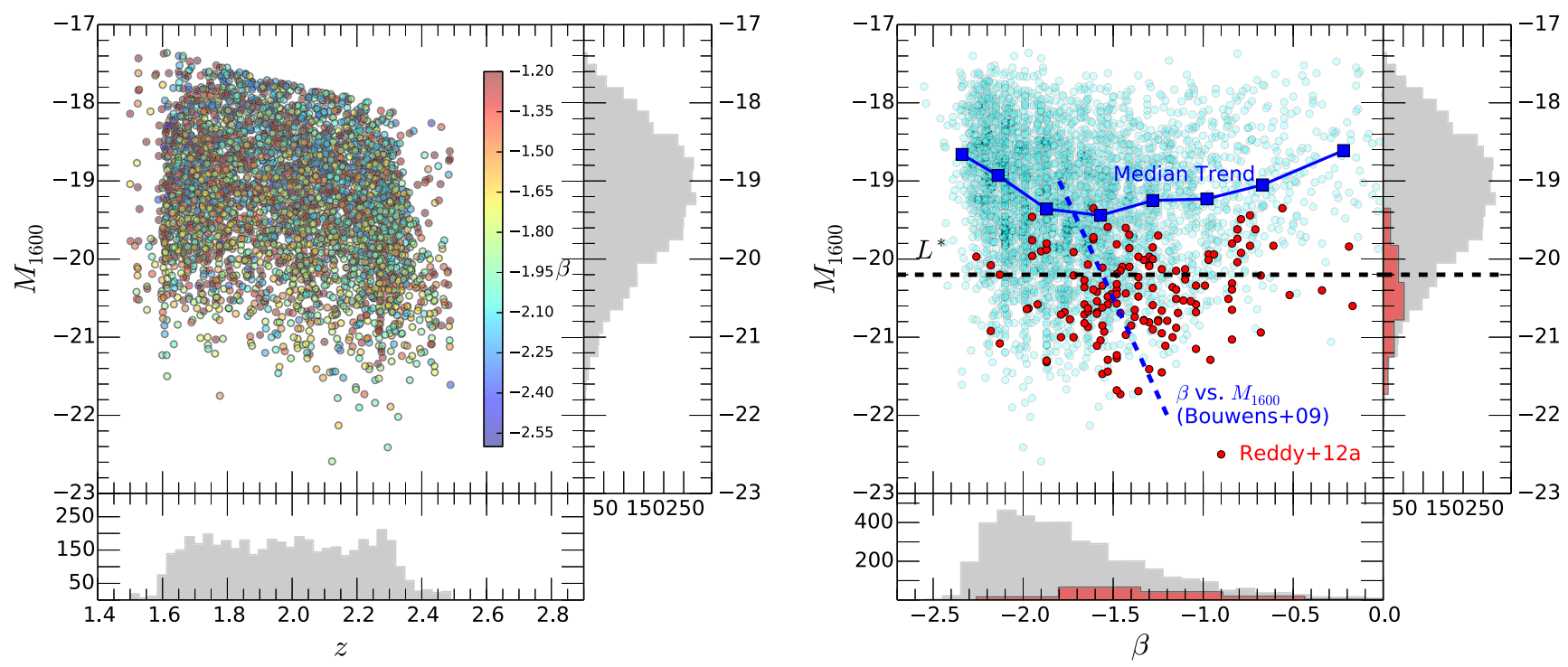

Figure 1. Redshift $(z)$, absolute magnitude $\left(M_{1600}\right)$, and UV slope $(\beta)$ distributions of the 3545 galaxies in our sample, color-coded by $\beta$ (left panel) and denoted by cyan symbols (right panel). For comparison, the distributions for the $124 \mathrm{UV}$-selected galaxies from Reddy et al. (2012a) are indicated by the red symbols and histograms in the right panel, while the black dashed line denotes the value of $M_{1500}^{*} \simeq-20.2$ at $z \sim 1.9$ from Oesch et al. (2010). The present HDUV+3D-HST sample is a factor of $\approx 30$ larger than that of Reddy et al. (2012a) and includes galaxies over broader ranges of $M_{1600}$ and $\beta$, particularly at fainter magnitudes and bluer UV slopes. The blue squares and solid line in the right panel indicate the median relationship between $\beta$ and $M_{1600}$ for our sample, compared with the mean relationship (blue dashed line) found for $168 U$-dropouts at $z \sim 2.5$ (note that this is the upper bound in redshift for our sample) from Bouwens et al. (2009). Removing the $m_{(1+z) \times 1700} \leqslant 27.0$ requirement imposed to construct our sample (Section 2) would allow for a larger number of faint galaxies with redder UV slopes to be selected, increasing the number density of objects in the upper right region of the right panel.

IRX. The corresponding weighted average fluxes in each band $\left(\left\langle f_{24}\right\rangle,\left\langle f_{100}\right\rangle\right.$, and $\left.\left\langle f_{160}\right\rangle\right)$, where the weights are $1 / L_{\mathrm{UV}}$, were computed by multiplying the stacked fluxes by the weighted average UV luminosity of galaxies in the stack. The measurement uncertainties of these fluxes were calculated as the $1 \sigma$ dispersion in the fluxes obtained by fitting PSFs at random positions in the stacked images, avoiding the stacked signal itself.

While stacking on residual images aids in minimizing the contribution of bright nearby objects to the stacked fluxes, this method will not account for objects that are blended with the galaxies of interest in the Herschel/PACS imaging. This presents a particular challenge in our case, where the galaxies are selected from $H S T$ photometry, as a single galaxy (e.g., as observed from the ground) may be resolved with $H S T$ into several subcomponents, each of which is of course unresolved in the Herschel imaging, but each of which will contribute to the stacked flux. Galaxies that are resolved into multiple subcomponents will contribute more than once to the stack, resulting in an overestimate of the stacked far-IR flux. This effect is compounded by that of separate galaxies contributing more than once to the stack if they happen to be blended at the Herschel/PACS resolution. This bias was quantified as follows.

For a given band, we used the PSF to generate $N$ galaxies, where $N$ is the number of galaxies in the stack, each having a flux equal to the weighted average flux of the stacked signal. These simulated galaxies were added to the residual image at locations that were shifted from those of the real galaxies by offsets $\delta x$ and $\delta y$ in the $x$ - and $y$-directions, respectively, where the offsets were chosen randomly. This ensures that the spatial distribution of the simulated galaxies is identical to that of the real galaxies. We then stacked at the locations of the simulated galaxies and compared the simulated and recovered stacked fluxes. This was done 100 times, each time with a different pair of (randomly chosen) $\delta x$ and $\delta y$. The average ratio of the simulated and recovered fluxes, or the bias factor, from these 100 simulations varied from $\approx 0.60$ to 0.90 , depending on the number of galaxies contributing to the stack and the particular band. These simulations were performed for every band and for every stack in our analysis, and the stacked fluxes of the galaxies in our sample were multiplied by the bias factors calculated from these simulations.

To further investigate this bias, we also stacked all galaxies in our sample that had no $H S T$-detected object within 3!"35, corresponding to the half-width at half-maximum of the Herschel/PACS $100 \mu \mathrm{m}$ PSF. While this criterion severely restricts the size of the sample to only 465 objects, it allowed us to verify the bias factors derived from our simulations. Stacking these 465 objects yielded weighted average fluxes at 24, 100, and $160 \mu \mathrm{m}$ that are within $1 \sigma$ of the values obtained for the entire sample once the bias factors are applied. ${ }^{13}$

Infrared luminosities were calculated by fitting the Elbaz et al. (2011) "main-sequence" dust template to the stacked $\left\langle f_{100}\right\rangle$ and $\left\langle f_{160}\right\rangle$ fluxes. We chose this particular template because it provided the best match to the observed infrared colors $f_{100} / f_{160}$ of the stacks, though we note that the adoption of other templates (e.g., Chary \& Elbaz 2001; Dale \& Helou 2002; Rieke et al. 2009) results in $L_{\mathrm{IR}}$ that vary by no more than $\approx 50 \%$ from the ones calculated here (see Reddy et al. 2012a, for a detailed comparison of $L_{\mathrm{IR}}$ computed using different dust templates). Upper limits in $L_{\mathrm{IR}}$ are quoted in cases where $L_{\mathrm{IR}}$ divided by the modeled uncertainty is $>3$. In a few instances, $\sim 2 \sigma$ detections of both the 100 and $160 \mu \mathrm{m}$

\footnotetext{
${ }^{13}$ While the $160 \mu \mathrm{m}$ PSF has a half-width at half-maximum that is larger than the exclusion radius of 3 !" 35 , the agreement in the average $f_{100} / f_{160}$ ratio, or far-infrared color, between the stack of the full sample and that of the 465 galaxies suggests that the bias factors also recover successfully the average $160 \mu \mathrm{m}$ stacked flux.
} 
Table 2

Stacked Fluxes and Infrared and UV Luminosities

\begin{tabular}{|c|c|c|c|c|c|c|c|c|c|}
\hline Sample & $N^{\mathrm{a}}$ & $\langle z\rangle^{\mathrm{b}}$ & $\langle\beta\rangle^{\mathrm{b}}$ & $\begin{array}{l}\left\langle f_{24}\right\rangle^{\mathrm{c}} \\
(\mu \mathrm{Jy})\end{array}$ & $\begin{array}{l}\left\langle f_{100}\right\rangle^{\mathrm{c}} \\
(\mu \mathrm{Jy})\end{array}$ & $\begin{array}{l}\left\langle f_{160}\right\rangle^{\mathrm{c}} \\
(\mu \mathrm{Jy})\end{array}$ & $\begin{array}{c}\left\langle L_{7.7}\right\rangle^{\mathrm{d}} \\
\left(10^{10} L_{\odot}\right)\end{array}$ & $\begin{array}{c}\left\langle L_{\mathrm{IR}}\right\rangle^{\mathrm{d}} \\
\left(10^{10} L_{\odot}\right)\end{array}$ & $\begin{array}{c}\left\langle L_{\mathrm{UV}}\right\rangle^{\mathrm{d}} \\
\left(10^{10} L_{\odot}\right)\end{array}$ \\
\hline All & 3545 & 1.94 & -1.71 & $1.54 \pm 0.14$ & $29 \pm 6$ & $62 \pm 17$ & $0.26 \pm 0.03$ & $2.1 \pm 0.4$ & 0.80 \\
\hline \multicolumn{10}{|l|}{$M_{1600}$ bins: } \\
\hline$M_{1600} \leqslant-21$ & 81 & 2.12 & -1.74 & $4.83 \pm 0.96$ & $177 \pm 30$ & $377 \pm 93$ & $1.00 \pm 0.20$ & $17.1 \pm 2.4$ & 6.73 \\
\hline$-21<M_{1600} \leqslant-20$ & 575 & 2.07 & -1.68 & $4.37 \pm 0.28$ & $87 \pm 13$ & $171 \pm 43$ & $0.86 \pm 0.06$ & $7.6 \pm 1.0$ & 2.92 \\
\hline$-20<M_{1600} \leqslant-19$ & 1390 & 1.99 & -1.67 & $2.33 \pm 0.20$ & $38 \pm 8$ & $84 \pm 25$ & $0.41 \pm 0.03$ & $3.1 \pm 0.6$ & 1.26 \\
\hline $\begin{array}{l}M_{1600}>-19 \\
\beta \text { bins: }\end{array}$ & 1499 & 1.92 & -1.72 & $1.00 \pm 0.16$ & $31 \pm 9$ & $54 \pm 24$ & $0.16 \pm 0.03$ & $2.0 \pm 0.5$ & 0.48 \\
\hline$\beta \leqslant-1.70$ & 2084 & 1.96 & -2.04 & $0.52 \pm 0.16$ & $5 \pm 7$ & $21 \pm 18$ & $0.09 \pm 0.03$ & $<1.4$ & 0.77 \\
\hline$-1.70<\beta \leqslant-1.40$ & 722 & 1.92 & -1.56 & $1.89 \pm 0.41$ & $43 \pm 13$ & $86 \pm 37$ & $0.31 \pm 0.07$ & $2.9 \pm 0.7$ & 0.95 \\
\hline$-1.40<\beta \leqslant-1.10$ & 345 & 1.94 & -1.26 & $3.92 \pm 0.55$ & $52 \pm 18$ & $103 \pm 56$ & $0.65 \pm 0.09$ & $3.7 \pm 1.1$ & 0.93 \\
\hline$-1.10<\beta \leqslant-0.80$ & 205 & 1.93 & -0.97 & $7.07 \pm 0.53$ & $80 \pm 25$ & $173 \pm 73$ & $1.15 \pm 0.09$ & $5.7 \pm 1.4$ & 0.81 \\
\hline$\beta>-0.80$ & 189 & 1.90 & -0.31 & $5.09 \pm 0.62$ & $167 \pm 23$ & $340 \pm 63$ & $0.80 \pm 0.10$ & $11.0 \pm 1.2$ & 0.59 \\
\hline \multicolumn{10}{|l|}{$M_{1600}$ and $\beta$ bins: } \\
\hline$M_{1600} \leqslant-19+\beta \leqslant-1.4$ & 1616 & 2.01 & -1.86 & $1.86 \pm 0.21$ & $25 \pm 9$ & $51 \pm 21$ & $0.33 \pm 0.04$ & $1.9 \pm 0.5$ & 1.58 \\
\hline$M_{1600} \leqslant-19+\beta>-1.4$ & 430 & 1.97 & -1.02 & $7.20 \pm 0.50$ & $117 \pm 19$ & $288 \pm 46$ & $1.25 \pm 0.09$ & $9.5 \pm 1.0$ & 1.47 \\
\hline$M_{1600}>-19+\beta \leqslant-1.4$ & 1190 & 1.92 & -1.97 & $0.36 \pm 0.21$ & $13 \pm 7$ & $26 \pm 23$ & $0.06 \pm 0.03$ & $<1.0$ & 0.48 \\
\hline$M_{1600}>-19+\beta>-1.4$ & 309 & 1.90 & -0.79 & $3.11 \pm 0.38$ & $95 \pm 19$ & $176 \pm 75$ & $0.49 \pm 0.06$ & $6.3 \pm 1.2$ & 0.48 \\
\hline \multicolumn{10}{|l|}{ Stellar mass and $\beta$ bins: } \\
\hline $\log \left[M_{*} / M_{\odot}\right] \leqslant 9.75$ & 2571 & 1.94 & -1.88 & $0.75 \pm 0.14$ & $10 \pm 7$ & $17 \pm 20$ & $0.13 \pm 0.03$ & $<1.2$ & 0.71 \\
\hline$+\beta \leqslant-1.4$ & 2385 & 1.94 & -1.95 & $0.63 \pm 0.19$ & $11 \pm 6$ & $28 \pm 15$ & $0.10 \pm 0.03$ & $<1.0$ & 0.72 \\
\hline$+\beta>-1.4$ & 186 & 1.89 & -1.12 & $2.95 \pm 0.76$ & $19 \pm 25$ & $72 \pm 73$ & $0.47 \pm 0.12$ & $<4.0$ & 0.57 \\
\hline $\log \left[M_{*} / M_{\odot}\right]>9.75$ & 974 & 1.96 & -0.92 & $4.93 \pm 0.40$ & $111 \pm 12$ & $229 \pm 36$ & $0.84 \pm 0.07$ & $8.3 \pm 0.7$ & 1.22 \\
\hline$+\beta \leqslant-1.4$ & 421 & 2.04 & -1.61 & $4.22 \pm 0.42$ & $59 \pm 14$ & $118 \pm 46$ & $0.80 \pm 0.07$ & $5.1 \pm 1.1$ & 2.26 \\
\hline$+\beta>-1.4$ & 553 & 1.94 & -0.72 & $5.23 \pm 0.48$ & $132 \pm 14$ & $263 \pm 44$ & $0.87 \pm 0.09$ & $9.4 \pm 0.8$ & 0.90 \\
\hline \multicolumn{10}{|l|}{ Age bins: } \\
\hline $\log [$ Age $/$ year $] \leqslant 8.00$ & 81 & 1.96 & -1.49 & $0.32 \pm 0.92$ & $62 \pm 39$ & $135 \pm 91$ & $<0.51$ & $<6.3$ & 0.55 \\
\hline $\log [$ Age $/$ year $]>8.00$ & 3464 & 1.94 & -1.71 & $1.43 \pm 0.22$ & $25 \pm 6$ & $52 \pm 19$ & $0.23 \pm 0.04$ & $1.8 \pm 0.4$ & 0.81 \\
\hline
\end{tabular}

Notes.

${ }^{a}$ Number of objects in the stack.

b Mean redshift and UV slope of objects in the stack.

${ }^{\mathrm{c}}$ Stacked 24, 100, and $160 \mu \mathrm{m}$ fluxes.

d Stacked $8 \mu \mathrm{m}$ and total infrared luminosities, and the mean UV luminosity of objects in the stack.

stacked fluxes yield a modeled $L_{\mathrm{IR}}$ that is significant at the $3 \sigma$ level.

The mean UV slope of objects contributing to the stack was computed as a weighted average of the UV slopes of individual objects where, again, the weights are $1 / L_{\mathrm{UV}}$. These same weights were also applied when calculating the weighted average redshift, absolute magnitude, stellar mass, and age of objects contributing to the stack. Table 2 lists the average galaxy properties and fluxes for each stack performed in our study.

\section{Predicted IRX $-\beta$ Relations}

We calculated the relationship between IRX and $\beta$ using the recently developed "Binary Population and Spectral Synthesis" (BPASS) models (Eldridge \& Stanway 2012; Stanway et al. 2016) with a stellar metallicity of $Z=0.14 Z_{\odot}$ on the current abundance scale (Asplund et al. 2009) and a twopower-law IMF with $\alpha=2.35$ for $M_{*}>0.5 M_{\odot}$ and $\alpha=1.30$ for $0.1 M_{\odot} \leqslant M_{*} \leqslant 0.5 M_{\odot}$. We assumed a constant star formation with an age of $100 \mathrm{Myr}$ and included nebular continuum emission. This particular BPASS model (what we refer to as our "fiducial" model) is found to best reproduce simultaneously the rest-frame far-UV continuum, stellar, and nebular lines and the rest-frame optical nebular emission line strengths measured for galaxies at $z \sim 2$ (Steidel et al. 2016). Two salient features of this model are the very blue intrinsic
UV continuum slope $\beta_{0} \simeq-2.62$ relative to that assumed in the Meurer et al. (1999) calibration of the IRX $-\beta$ relation $\left(\beta_{0}=-2.23\right)$ and the larger number of ionizing photons per unit SFR (i.e., $\approx 20 \%$ larger than those of single-star models with no stellar rotation; Stanway et al. 2016) that are potentially available for heating dust. For comparison, the BPASS model for the same metallicity with a constant star formation history and an age of $300 \mathrm{Myr}$ (the median for the sample considered here, and similar to the mean age of $z \sim 2 \mathrm{UV}$-selected galaxies; Reddy et al. 2012b) is $\beta_{0}=-2.52$. Below, we also consider the more traditionally used Bruzual \& Charlot (2003) (BC03) models.

We calculated the IRX $-\beta$ relation assuming an energy balance between flux that is absorbed and that which is reemitted in the infrared (Meurer et al. 1999). The absorption is determined by the extinction or attenuation curve, and we considered several choices, including the SMC extinction curve of Gordon et al. (2003) and the Calzetti et al. (2000) and Reddy et al. (2015) attenuation curves. The original forms of these extinction/attenuation curves were empirically calibrated at $\lambda \gtrsim 1200 \AA$. The Calzetti et al. (2000) and Reddy et al. (2015) curves were extended down to $\lambda=950 \AA$ using a large sample of LBG spectra at $z \sim 3$ and a newly developed iterative method presented in Reddy et al. (2016a). The SMC curve of Gordon et al. (2003) was extended in the same way, and we used these extended versions of the curves in this analysis. For 
reference, our new constraints on the shape of dust obscuration curves imply a lower attenuation of $\lambda \lesssim 1250 \AA$ photons relative to that predicted from polynomial extrapolations below these wavelengths (Reddy et al. 2016a). In practice, because most of the dust heating arises from photons with $\lambda>1200 \AA$, the implementation of the new shapes of extinction/attenuation curves does little to alter the predicted IRX $-\beta$ relation. For reference, the following equations give the relationship between $E(B-V)$ and $\beta$ for the fiducial (BPASS) model with nebular continuum emission and the shapes of the attenuation/ extinction curves derived above:

$$
\begin{aligned}
\text { Calzetti }+00: \beta & =-2.616+4.684 \times E(B-V) ; \\
\text { SMC }: \beta & =-2.616+11.259 \times E(B-V) ; \\
\text { Reddy }+15: \beta & =-2.616+4.594 \times E(B-V) .
\end{aligned}
$$

The intercepts in the above equations are equal to -2.520 for the 300 Myr BPASS model.

For each value of $E(B-V)$, we applied the aforementioned dust curves to the BPASS model and calculated the flux absorbed at $\lambda>912 \AA$. Based on the high covering fraction $(\gtrsim 92 \%)$ of optically thick $\mathrm{HI}$ inferred for $z \sim 3$ galaxies (Reddy et al. 2016b), we assumed a zero escape fraction of ionizing photons and that photoelectric absorption dominates the depletion of such photons, rather than dust attenuation (Reddy et al. 2016b). We then calculated the resultant Ly $\alpha$ flux assuming Case B recombination and the amount of $\operatorname{Ly} \alpha$ flux absorbed given the value of the extinction/attenuation curve at $\lambda=1216 \AA$, and we added this to the absorbed flux at $\lambda>912 \AA$. This total absorbed flux is equated to $L_{\mathrm{IR}}$, where we have assumed that all of the dust emission occurs between 8 and $1000 \mu \mathrm{m}$.

Finally, we divided the infrared luminosity by the UV luminosity of the reddened model at $1600 \AA$ to arrive at the value of IRX. The UV slope was computed directly from the reddened model using the full set of Calzetti et al. (1994) wavelength windows. Below, we also consider the value of $\beta$ computed using the subset of the Calzetti et al. (1994) windows at $\lambda<1740 \AA$, as well as a single window spanning the range 1300-1800 A. Formally, we find the following relations between IRX and $\beta$ given Equation (1), where $\beta$ is measured using the full set of Calzetti et al. (1994) wavelength windows:

$$
\begin{aligned}
& \text { Calzetti }+00: \text { IRX }=1.67 \times\left[10^{0.4(2.13 \beta+5.57)}-1\right] ; \\
& \text { SMC }: \text { IRX }=1.79 \times\left[10^{0.4(1.07 \beta+2.79)}-1\right] ; \\
& \text { Reddy }+15: \operatorname{IRX}=1.68 \times\left[10^{0.4(1.82 \beta+4.77)}-1\right] .
\end{aligned}
$$

These relations may be shifted redder by $\delta \beta=0.096$ to reproduce the IRX $-\beta$ relations for the 300 Myr BPASS model. For reference, Appendix A summarizes the relations between $\beta$ and $E(B-V)$ and between IRX and $\beta$ for different assumptions of the stellar population model, nebular continuum, Ly $\alpha$ heating, and normalization of the dust curve.

Figures 2 and 3 convey a sense for how the stellar population and nebular continuum, Ly $\alpha$ heating, UV slope measurements, and total-to-selective extinction $\left(R_{\mathrm{V}}\right)$ affect the IRX $-\beta$ relation. Models with a bluer intrinsic UV slope require a larger degree of dust obscuration to reproduce a given observed UV slope, thus causing the IRX $-\beta$ relation to shift toward bluer $\beta$. Relative to the Meurer et al. (1999) relation, the IRX $-\beta$ relations for the fiducial (BPASS) 100 and 300 Myr models predict a factor of $\approx 2$ more dust obscuration at a given $\beta$ for $\beta \gtrsim-1.7$, and an even larger factor for $\beta$ bluer than this limit (left panel of Figure 2). The commonly utilized BC03 model results in a factor of $\approx 30 \%$ increase in the IRX at a given $\beta$ relative to the Meurer et al. (1999) curve, while the $0.28 Z_{\odot}$ $\mathrm{BC} 03$ model results in an IRX $-\beta$ relation that is indistinguishable from that of the BPASS model for the same age (right panel of Figure 3). These predictions underscore the importance of the adopted stellar population model when using the IRX $-\beta$ relation to discern between different dust attenuation/ extinction curves (e.g., Meurer et al. 1999; Boquien et al. 2012; Schaerer et al. 2013). Note that the inclusion of nebular continuum emission shifts the IRX $-\beta$ relation by $\delta \beta \simeq 0.1$ to the right (i.e., making $\beta$ redder), so that the IRX at a given $\beta$ is $\approx 0.1$ dex lower (leftmost panel of Figure 3).

The specific treatment of dust heating from Ly $\alpha$ photons has a much less pronounced effect on the IRX $-\beta$ relation. If none of the Ly $\alpha$ flux is absorbed by dust-also equivalent to assuming that the escape fraction of ionizing photons is $100 \%$ - then the resulting IRX is $\approx 10 \%$ lower at a given $\beta$ than that predicted by our fiducial model. Similarly, assuming that all of the Ly $\alpha$ is absorbed by dust results in an IRX $-\beta$ relation that is indistinguishable from that of the fiducial model.

The wavelengths over which $\beta$ is computed will also effect the IRX $-\beta$ relation to varying degrees, depending on the specific wavelength ranges and the stellar population model. For the BPASS model, computing $\beta$ from the reddened model spectrum within a single window spanning the range $1300-1800 \AA$ results in an IRX $-\beta$ relation that is shifted by as much as $\delta \beta=0.4$ to redder slopes. This effect is due to the fact that the stellar continuum rises less steeply toward shorter wavelengths for $\lambda \lesssim 1500 \AA$. Consequently, the $\log ($ IRX) is $\simeq 0.18$ dex lower in this case relative to that computed based on the full set of Calzetti et al. (1994) windows. Similar offsets are observed when using the subset of the Calzetti et al. (1994) windows lying at $\lambda<1800 \AA$, while the offsets are not as large with the BC03 model. Most previous studies of the IRX $-\beta$ relation adopted a $\beta$ computed over relatively broad wavelength ranges coinciding with the Calzetti et al. (1994) windows. However, the systematic offsets in the IRX $-\beta$ relation arising from the narrower wavelength range used to compute UV slopes become relevant for very high redshift (e.g., $z \gtrsim 8$ ) galaxies, where Hubble photometry is typically used to constrain the UV slope and where such observations only go as red as rest-frame $\lesssim 1800 \AA$.

Finally, the rightmost panel of Figure 3 shows the effect of lowering the total-to-selective extinction $\left(R_{\mathrm{V}}\right)$, or normalization, of the attenuation/extinction curves by various amounts.

Of the physical factors discussed above, the IRX $-\beta$ relation is most sensitive to the effects of changing the intrinsic UV slope and $/$ or $R_{\mathrm{V}}$. To underscore the importance of the assumed stellar population when interpreting the IRX $-\beta$ relation, we show in Figure 4 the comparison of our fiducial BPASS model assuming the Calzetti et al. (2000) curve and an intrinsic $\beta_{0}=-2.23$ (accomplished by simply shifting the model to asymptote to this intrinsic value), along with the same model assuming an SMC curve with $\beta_{0}=-2.62$. As is evident from this figure, the two IRX $-\beta$ relations that assume different attenuation curves and intrinsic UV slopes have a significant overlap (within a factor of two in IRX) over the range $-2.1 \lesssim \beta \lesssim-1.3$. Notably this range includes the typical $\beta \simeq-1.5$ found for UV-selected galaxies at $z \sim 2$ (see Reddy et al. 2012a). In the next section, we examine these effects 


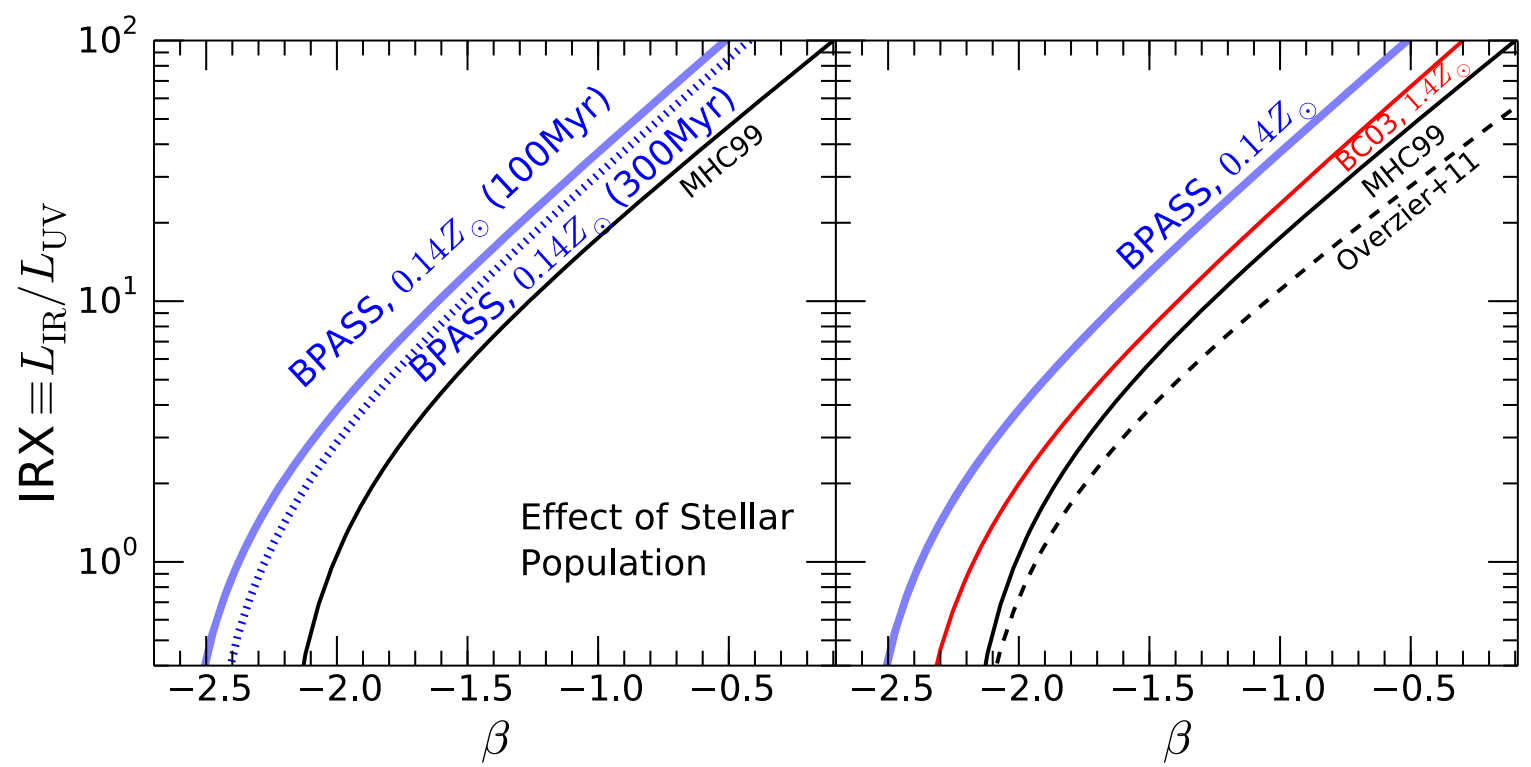

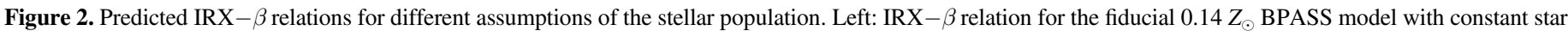

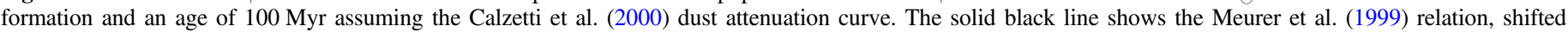

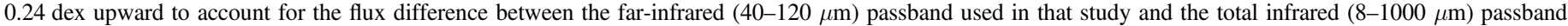

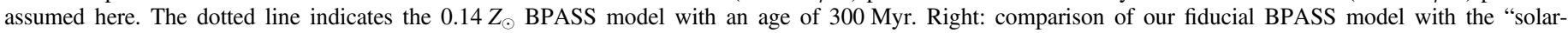

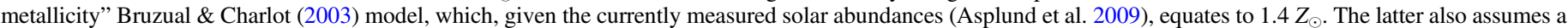

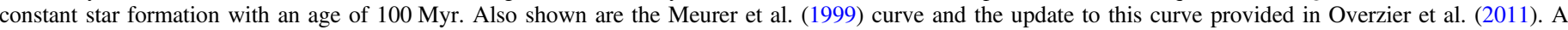

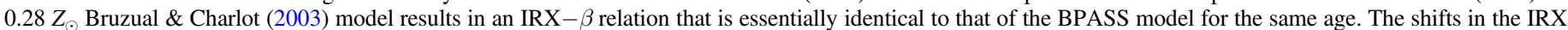

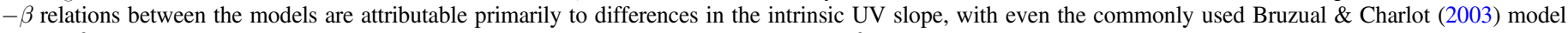
having $\beta_{0}=-2.44$ (without including nebular continuum), substantially bluer than the $\beta_{0}=-2.23$ model adopted in Meurer et al. (1999).

further in the context of the stacked constraints on IRX $-\beta$ provided by the combined HDUV and 3D-HST samples.

\section{Discussion}

\subsection{IRX- $\beta$ for the Entire Sample}

As a first step in constraining the IRX $-\beta$ relation at $z=1.5-2.5$, we stacked galaxies in bins of UV slope. The resulting IRX for each of these bins, as well as for the whole sample, is shown in Figure 5. The predicted IRX $-\beta$ relations for different assumptions of the stellar population (BPASS or $\mathrm{BC} 03$ ) intrinsic UV slope, $\beta_{0}$, and the difference in normalization of the dust curves, $\delta R_{\mathrm{V}}$, are also shown. To account for the former, we simply shifted the fiducial relation (computed assuming $\beta_{0}=-2.62$ ) so that it asymptotes to a redder value of $\beta_{0}=-2.23$, similar to that assumed in Meurer et al. (1999).

Recently, McLure et al. (2017) have pointed out that, for sufficiently large errors in the UV slope, there can be a significant scattering of objects from bins of bluer $\beta$ to bins of redder $\beta$ given that generally there are many more bluer objects than redder ones in a UV-selected sample. The result is that estimates of IRX in bins of observed $\beta$ can be lower than the intrinsic values, leading one to measure a shallower slope of the IRX $-\beta$ relation. To examine the effect of this bias on our sample, we performed a simulation similar to that outlined in McLure et al. (2017). Specifically, we simulated a population of galaxies with a Schechter (1976) stellar mass function with $\log \left[\phi / \mathrm{Mpc}^{-3}\right]=-3.5, \log \left[M_{*} / M_{\odot}\right]=10.6$, and $\alpha=-1.6$ (Davidzon et al. 2017). Each galaxy was assigned a value of IRX based on the "consensus" relation between IRX and $M_{*}$ at $z \sim 2$ (Bouwens et al. 2016a), randomly perturbed by assuming a scatter in the IRX $-M_{*}$ relation of 0.3 dex. Each value of IRX corresponds to a specific UV slope for a given intrinsic UV slope $\left(\beta_{0}\right)$ and a shape of the attenuation curve.
We assigned each galaxy a $\beta$ based on assuming $\beta_{0}=-2.62$ and the Calzetti et al. (2000) attenuation curve. Each $\beta$ was perturbed assuming a $\sigma_{\beta}=0.19$, the typical error in the photometrically determined $\beta$ (Section 2). The galaxies were then binned according to their unperturbed and perturbed $\beta$ values, and the average IRX values were computed in these bins. According to this simulation, the average IRX in the bins of perturbed $\beta$ were within $10 \%(<0.04 \mathrm{dex})$ of the average IRX in bins of the unperturbed $\beta$. This systematic uncertainty is substantially smaller than the typical random uncertainty in $\log [$ IRX $]$ of $\simeq 0.10$ dex. Thus, we conclude that uncertainties in $\beta$ for the HDUV sample are insufficient to significantly bias our determination of the IRX $-\beta$ relation. Moreover, our stack of the entire sample, as well as the stacks in bins of stellar mass (Section 5.2), are unaffected by the aforementioned bias.

Our stacked results indicate a highly significant $(\gtrsim 20 \sigma)$ correlation between IRX and $\beta$. However, none of the predicted relations calculated based on assuming an intrinsic UV slope of $\beta_{0}=-2.23$, as in Meurer et al. (1999), are able to reproduce our stacked estimates for the full range of $\beta$ considered here. For example, the top left panel of Figure 5 shows that while both the Calzetti et al. (2000) and Reddy et al. (2015) attenuation curves predict IRX that are within $3 \sigma$ of our stacked values for $\beta<-1.2$, they overpredict the IRX for galaxies with redder $\beta$.

Lowering the normalization of the Reddy et al. (2015) attenuation curve by $\delta R_{\mathrm{V}}=1.5$ results in a better match to the stacked determinations, but with some disagreement (at the $>3 \sigma$ level) with the stack of the entire sample (bottom left panel of Figure 5). Reddy et al. (2015) estimated the systematic uncertainty in their determination of $R_{\mathrm{V}}$ to be $\delta R_{\mathrm{V}} \approx 0.4$, which suggests that their curve may not have a normalization as low as $R_{\mathrm{V}}=1.0$ given their favored value of $R_{\mathrm{V}}=2.51$. Regardless, without any modifications to the normalizations 


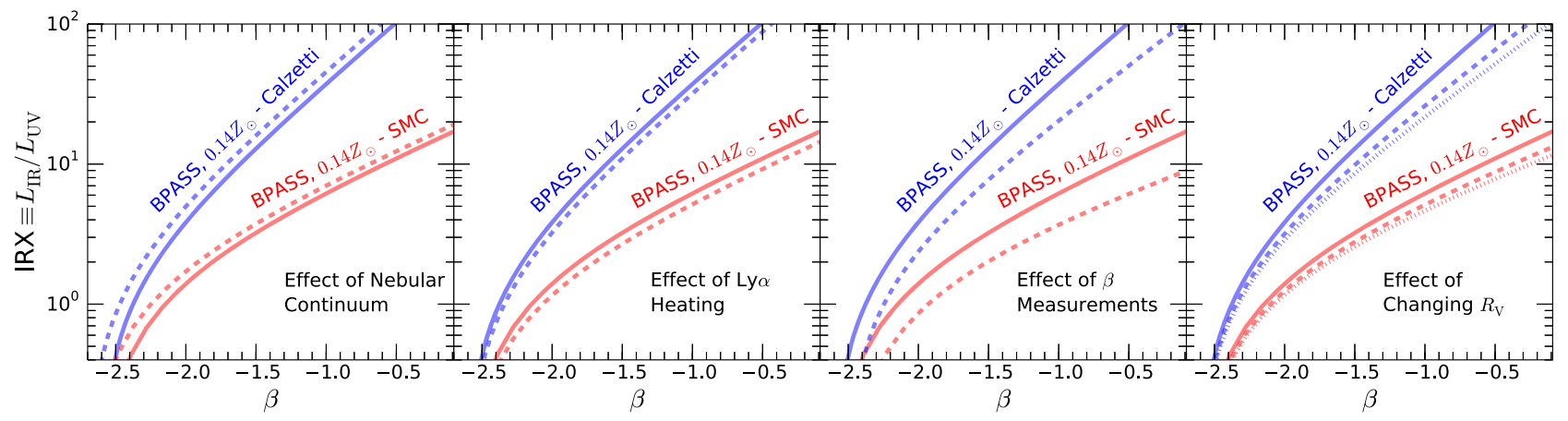

Figure 3. Predicted IRX $-\beta$ relations for different assumptions of the contribution of nebular continuum emission, effect of Ly $\alpha$ heating, systematics associated with the measurement of the UV slope, and the normalization of the dust curves. Left: IRX $-\beta$ relations for the fiducial $0.14 Z_{\odot}$ BPASS model, with (solid line) and without (dashed line) including nebular continuum emission. Relations are show for both the Calzetti et al. (2000) and SMC dust curves. Neglecting the contribution to the SED from nebular continuum emission will cause one to measure a slightly bluer UV slope $(\delta \beta \simeq 0.1)$. Middle left: IRX $-\beta$ relations for the fiducial model, assuming the Calzetti et al. (2000) attenuation and the SMC extinction curves (solid lines). The dashed lines indicate the result if we assume that none of the Ly $\alpha$ emission from the galaxy is absorbed by dust. Middle right: same as the middle left panel, but the dashed lines now indicate the IRX $-\beta$ relations if $\beta$ is measured using a window spanning the range 1300-1800 А. Right: same as the middle left panel, but the dashed and dotted lines now show the effect of lowering the total-to-selective extinction by $\delta R_{\mathrm{V}}=1.0$ and 1.5 , respectively.

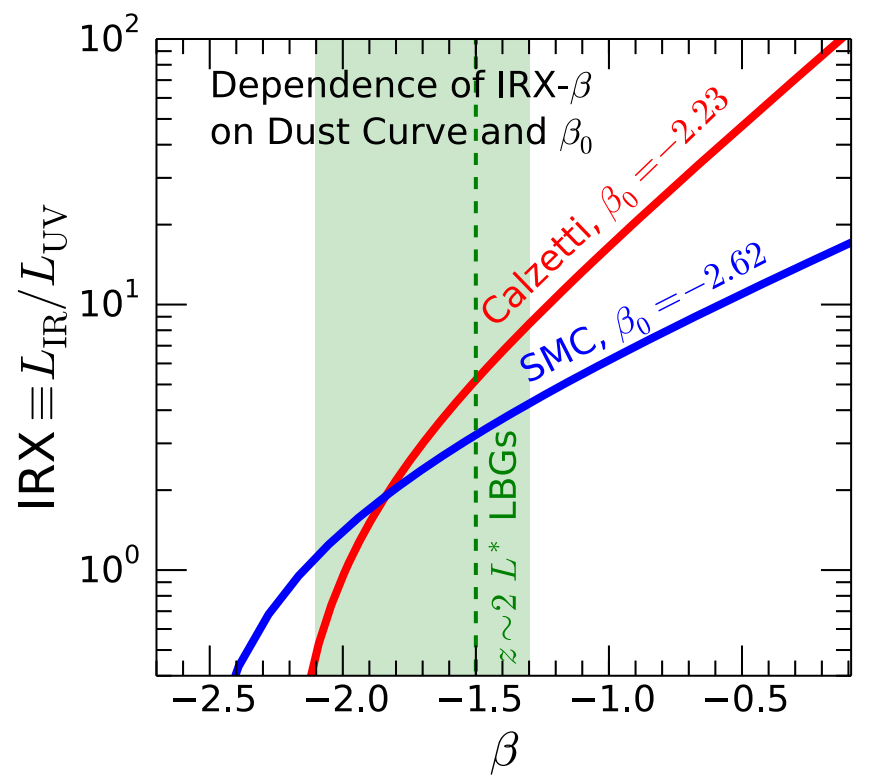

Figure 4. Predicted IRX $-\beta$ relation for our fiducial model, shifted to have an intrinsic UV slope of $\beta_{0}=-2.23$, assuming the Calzetti et al. (2000) dust attenuation curve (red line). The blue curve shows the fiducial model when assuming $\beta_{0}=-2.62$ and the SMC curve. There is a substantial overlap (within an factor of two in IRX) between the two IRX $-\beta$ relations over the range $-2.1 \lesssim \beta \lesssim-1.3$ (light-green shaded region), where $L^{*}$ LBGs at $z \sim 2-3$ tend to lie (vertical dashed line; Reddy et al. 2012a).

and/or shapes of the attenuation curves in the literature (Calzetti et al. 2000; Gordon et al. 2003; Reddy et al. 2015), the corresponding IRX $-\beta$ relations are unable to reproduce our stacked estimates if we assume an intrinsic UV slope of $\beta_{0}=-2.23$. At face value, these results suggest that the attenuation curve describing our sample is steeper than the typically utilized Calzetti et al. (2000) relation, but grayer than the SMC extinction curve. However, this conclusion depends on the intrinsic UV slope of the stellar population, as we discuss next.

Independent evidence favors the low-metallicity BPASS model in describing the underlying stellar populations of $z \sim 2$ galaxies (Steidel et al. 2016). The very blue intrinsic UV slope characteristic of this model-as well as those of the $\mathrm{BCO} 3$ models with comparable stellar metallicities (e.g., the $0.28 Z_{\odot}$
BC03 model with the same high-mass power-law index of the IMF as the BPASS model has $\beta_{0}=-2.65$ ) - is also favored in light of the non-negligible number of galaxies in our sample $(\approx 9 \%)$ that have $\beta<-2.23$ at the $3 \sigma$ level, the canonical value assumed in Meurer et al. (1999). Figure 2 shows that the low-metallicity models with blue $\beta_{0}$ result in IRX $-\beta$ relations that are significantly shifted relative to those assuming redder $\beta_{0}$. With such models, we find that our stacked measurements are best reproduced by an SMC-like extinction curve (top right panel of Figure 5), in the sense that all of the measurements lie within $3 \sigma$ of the associated prediction. On the other hand, with such stellar population models, grayer attenuation curves (e.g., Calzetti et al. 2000) overpredict the IRX at a given $\beta$ by a factor of $\approx 2-7$. More generally, we find that the slope of the IRX $-\beta$ relation implied by our stacked measurements is better fit with that obtained when considering the SMC extinction curve, while grayer attenuation curves lead to a more rapid rise in IRX with increasing $\beta$.

Our stacked measurements and predicted IRX $-\beta$ curves are compared with several results from the literature in Figure 6. In the context of the IRX $-\beta$ predictions that adopt subsolar metallicities, we find that most of the stacked measurements for UV-selected galaxies at $z \sim 1.5-3.0$ suggest a curve that is SMC-like, at least for $\beta \lesssim-0.5$. Several of the samples, including those of Heinis et al. (2013), Álvarez-Márquez et al. (2016), and Sklias et al. (2014), indicate an IRX that is larger than the SMC prediction for $\beta \gtrsim-0.5$. Such a behavior is not surprising given that the dust obscuration has been shown to decouple from the UV slope for galaxies with large SFRs, as is predominantly the case for most star-forming galaxies with very red $\beta$ (Goldader et al. 2002; Chapman et al. 2005; Reddy et al. 2006b, 2010; Penner et al. 2012; Casey et al. 2014b; Salmon et al. 2016).

As discussed in a number of studies (Reddy et al. 2006b, 2010; Penner et al. 2012; Casey et al. 2014b; Koprowski et al. 2016), dusty galaxies in general can exhibit a wide range in $\beta$ (from very blue to very red) depending on the particular spatial configuration of the dust and UV-emitting stars. Figure 6 shows that the degree to which such galaxies diverge from a given attenuation curve depends on $\beta_{0}$. Many of the dusty galaxies that would appear to have IRX larger than the Meurer et al. (1999) or Calzetti et al. (2000) predictions may in fact be adequately described by such curves if the stellar 


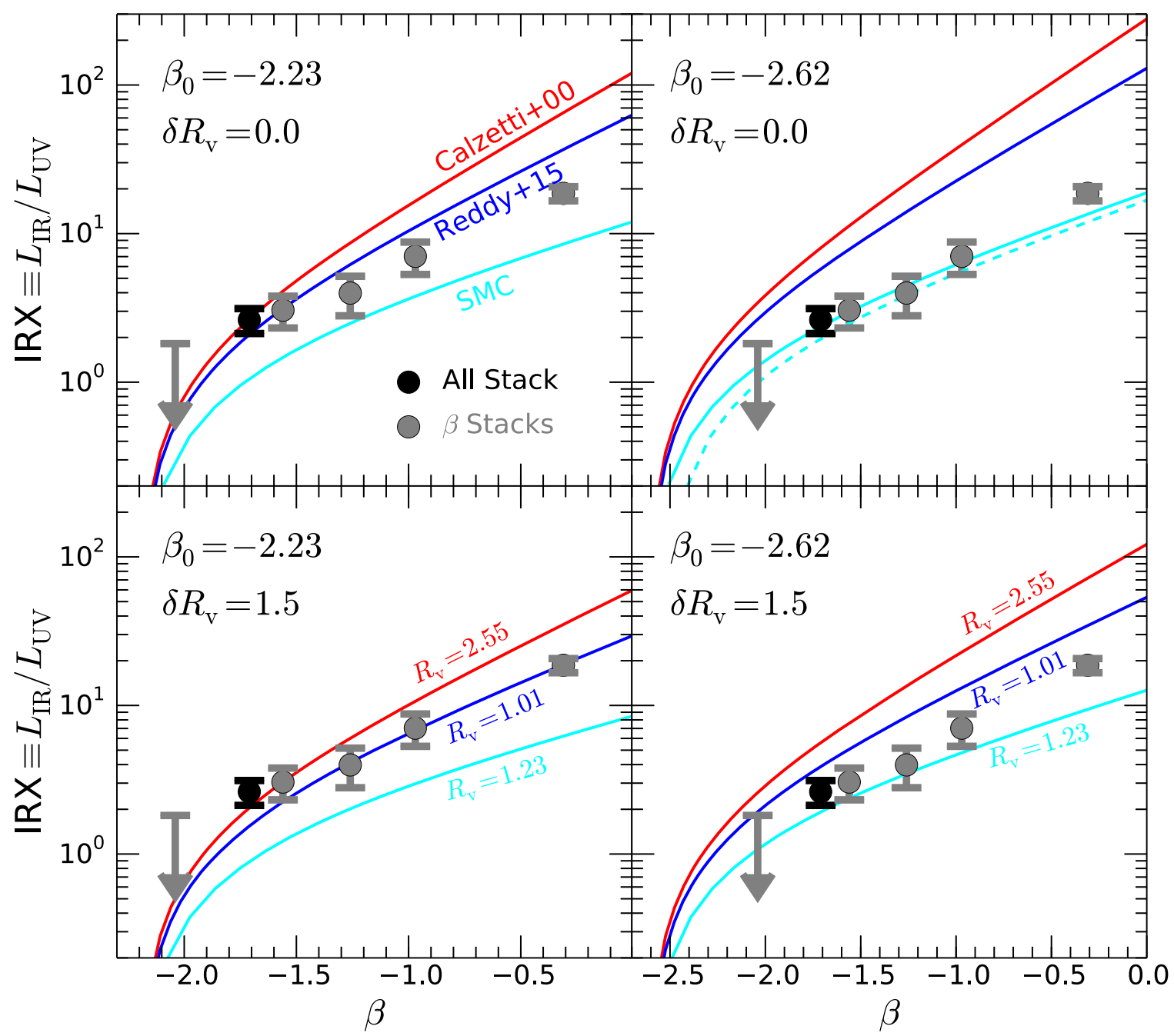

Figure 5. Predicted IRX $-\beta$ relations assuming the Calzetti et al. (2000), Reddy et al. (2015), and SMC dust curves-the Overzier et al. (2011) IRX - $\beta$ relation is indistinguishable from that obtained from Reddy et al. (2015) attenuation curve - for the fiducial stellar population model (BPASS). The intrinsic slope of this model is $\beta_{0}=-2.62$. In the left panels, the predicted IRX $-\beta$ relations have been shifted to show the effect of assuming a redder intrinsic UV slope of $\beta_{0}=-2.23$, the same as that in Meurer et al. (1999). The two bottom panels show the effect of lowering the normalization of the dust curves by $\delta R_{\mathrm{V}}=1.5$, with the specific values of $R_{\mathrm{v}}$ indicated. The gray points in each panel denote our stacked measurements of IRX for galaxies in bins of $\beta$, while the black points show the result of the stack for all galaxies. The dashed line in the top right panel indicates the IRX $-\beta$ relation implied by the SMC extinction curve for an age of $300 \mathrm{Myr}$.

populations of these galaxies are characterized by very blue intrinsic UV spectral slopes. On the other hand, if these dusty galaxies have relatively enriched stellar populations and redder intrinsic slopes, then their departure from the Calzetti et al. (2000) prediction would be enhanced.

Undoubtedly, large variations in IRX can also be expected with different geometries of dust and stars. Regardless, if subsolar-metallicity models are widely representative of the stellar populations of typical star-forming galaxies at $z \gtrsim 1.5$, then our stacked measurements, along with those in the literature, tend to disfavor gray attenuation curves for these galaxies. The large sample studied here, as well as those of Bouwens et al. (2016a) and Álvarez-Márquez et al. (2016), suggest an SMC-like curve. At first glance, this conclusion may appear to be at odds with the wide number of previous investigations that have found that the Meurer et al. (1999) and Calzetti et al. (2000) attenuation curves generally apply to moderately reddened star-forming galaxies at $z \gtrsim 1.5$ (e.g., Nandra et al. 2002; Seibert et al. 2002; Reddy \& Steidel 2004; Reddy et al. 2006b, 2010, 2012a; Daddi et al. 2007;
Pannella et al. 2009; Magdis et al. 2010; Overzier et al. 2011; De Barros et al. 2016; Forrest et al. 2016; see also Heinis et al. 2013).

In the framework of our present analysis, the reconciliation between these results is simple. Namely, our analysis does not call into question previous measurements of IRX $-\beta$, but calls for a different interpretation of these measurements. In the previous interpretation, most of the stacked measurements from the literature were found to generally agree with the Meurer et al. (1999) relation if we assume a relatively red intrinsic slope of $\beta=-2.23$. In the present interpretation, we argue that subsolar-metallicity models necessitate a steeper attenuation curve in order to reproduce the measurements of IRX - $\beta$ (e.g., see also Cullen et al. 2017). Our conclusion is aided by the larger dynamic range in $\beta$ probed by the HDUV and 3D-HST samples, which allows us to better discriminate between different curves given that their corresponding IRX $-\beta$ relations separate significantly at redder $\beta$ (Figures 4 and 6). 


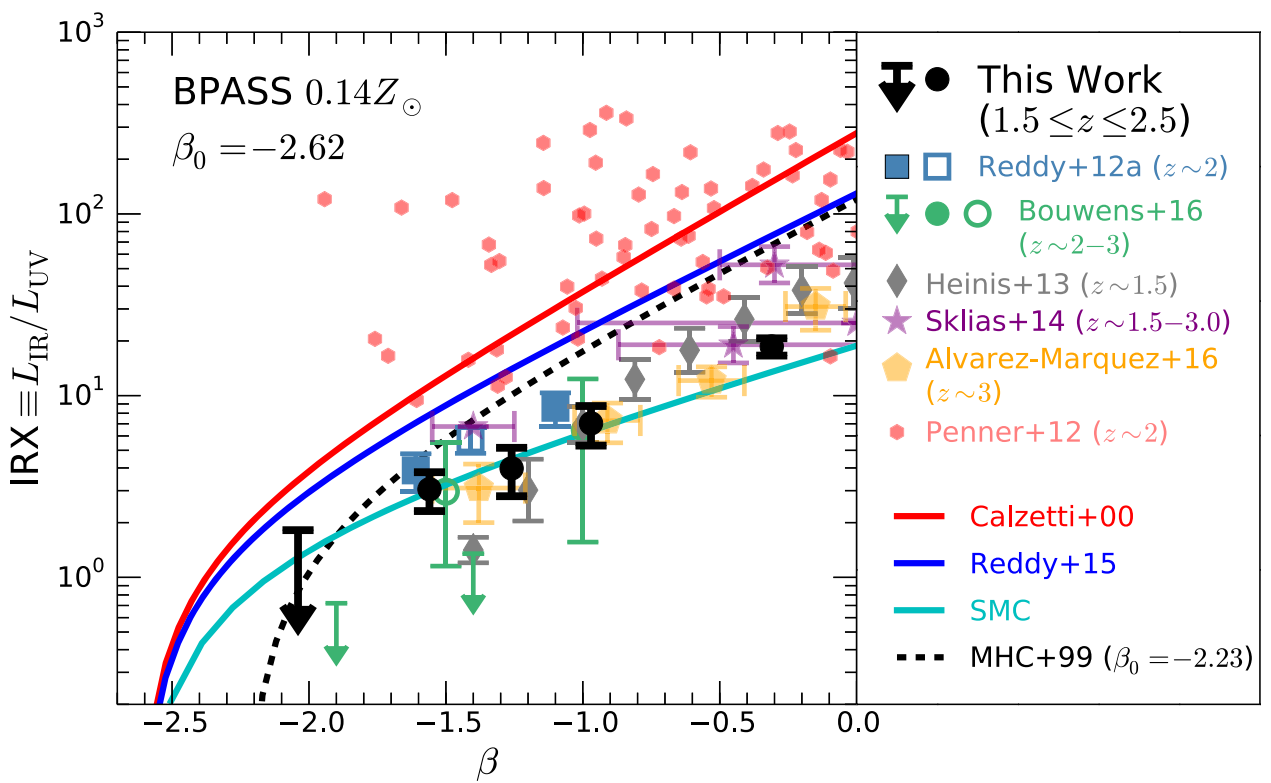

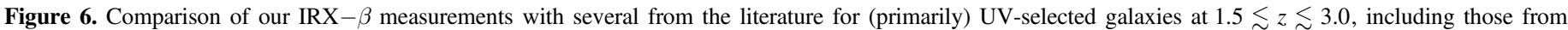

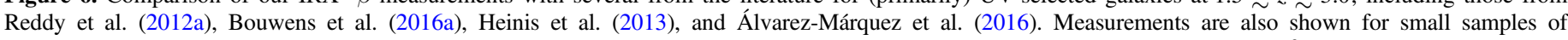

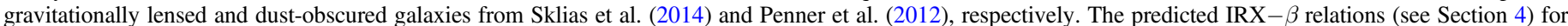

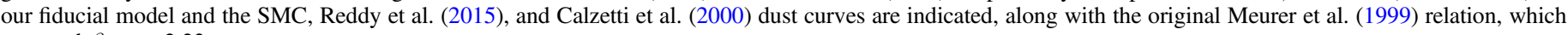
assumed $\beta_{0}=-2.23$.

\subsection{IRX versus Stellar Mass}

The well-studied correlations between SFR and stellar mass (e.g., Reddy et al. 2006b, 2012b; Daddi et al. 2007; Noeske et al. 2007; Pannella et al. 2009; Wuyts et al. 2011; Whitaker et al. 2014; Schreiber et al. 2015; Shivaei et al. 2015) and between SFR and dust attenuation (e.g., Wang \& Heckman 1996; Adelberger \& Steidel 2000; Reddy et al. 2006a, 2010; Buat et al. 2007, 2009; Burgarella et al. 2009) have motivated the use of stellar mass as a proxy for attenuation (Pannella et al. 2009, 2015; Reddy et al. 2010; Whitaker et al. 2014; Álvarez-Márquez et al. 2016; Bouwens et al. 2016a), as the former can be easily inferred from fitting stellar population models to broadband photometry. The connection between reddening and stellar mass can also be deduced from the mass-metallicity relation (Tremonti et al. 2004; Erb et al. 2006; Kewley \& Ellison 2008; Maiolino et al. 2008; Andrews \& Martini 2013; Henry et al. 2013; Maseda et al. 2014; Steidel et al. 2014; Sanders et al. 2015).

Motivated by these results, we stacked galaxies in two bins of stellar mass divided at $M_{*}=10^{9.75} M_{\odot}$ (and further subdivided into bins of $\beta$; Table 2) to investigate the dependence of the IRX $-\beta$ relation on stellar mass. ${ }^{14}$ The high-mass subsample $\left(M_{*}>10^{9.75} M_{\odot}\right)$ exhibits a redder UV slope $(\langle\beta\rangle=-0.92)$ and larger $\operatorname{IRX}(\langle\operatorname{IRX}\rangle=6.8 \pm 0.6)$ than the low-mass subsample with $\langle\beta\rangle=-1.88$ and $\langle$ IRX $\rangle<1.7$ ( $3 \sigma$ upper limit). Moreover, the high-mass subsample exhibits an IRX $-\beta$ relation consistent with that predicted assuming our fiducial stellar population model and the SMC extinction curve (Figure 7). Separately, the low-mass subsample as a whole, as well as the subset of the low-mass galaxies with $\beta \leqslant-1.4$, has $3 \sigma$ upper limits on IRX that require a dust curve that is at least as steep as the SMC.

\footnotetext{
14 The stellar masses obtained with Conroy \& Gunn (2010) models (Section 2) are on average within 0.1 dex of those obtained assuming the fiducial (BPASS) model with the same (Chabrier 2003) IMF.
}

The constraints on the IRX $-M_{*}$ relation from our sample are shown relative to previous determinations in the right panel of Figure 7. The " $z \sim 2$ Consensus Relation" presented in Bouwens et al. (2016a) was based on the IRX $-M_{*}$ trends published in Reddy et al. (2010), Whitaker et al. (2014), and Álvarez-Márquez et al. (2016). Formally, our stacked detection for the high-mass $\left(M_{*}>10^{9.75} M_{\odot}\right)$ subsample lies $\approx 4 \sigma$ below the consensus relation but is in excellent agreement with the mean IRX found for galaxies of similar masses $\left(\simeq 2 \times 10^{10} M_{\odot}\right)$ in Reddy et al. (2010). The upper limit in IRX for the low-mass $\left(M_{*} \leqslant 10^{9.75} M_{\odot}\right)$ sample is consistent with the predictions from the consensus relation. Based on these comparisons, we conclude that the IRX $-M_{*}$ relation from the present work is in general agreement with previous determinations and lends support for previous suggestions that stellar mass may be used as a rough proxy for dust attenuation in high-redshift star-forming galaxies (e.g., Pannella et al. 2009; Reddy et al. 2010; Bouwens et al. 2016a). Moreover, these comparisons underscore the general agreement between our IRX measurements (e.g., as a function of $\beta$ and $M_{*}$ ) and those in the literature, in spite of our different interpretation of these results in the context of the dust obscuration curve applicable to high-redshift galaxies (Section 5.1).

\subsection{IRX versus UV Luminosity}

As alluded to in Section 1, quantifying the dust attenuation of UV-faint (sub- $L^{*}$ ) galaxies has been a long-standing focus of the high-redshift community. While the steep faint-end slopes of UV luminosity functions at $z \gtrsim 2$ imply that such galaxies dominate the UV luminosity density at these redshifts, knowledge of their dust obscuration is required to assess their contribution to the cosmic SFR density (e.g., Steidel et al. 1999; Adelberger \& Steidel 2000; Bouwens et al. 2007; Reddy et al. 2008). Several studies have argued that UV-faint galaxies are on average less dusty than their brighter 


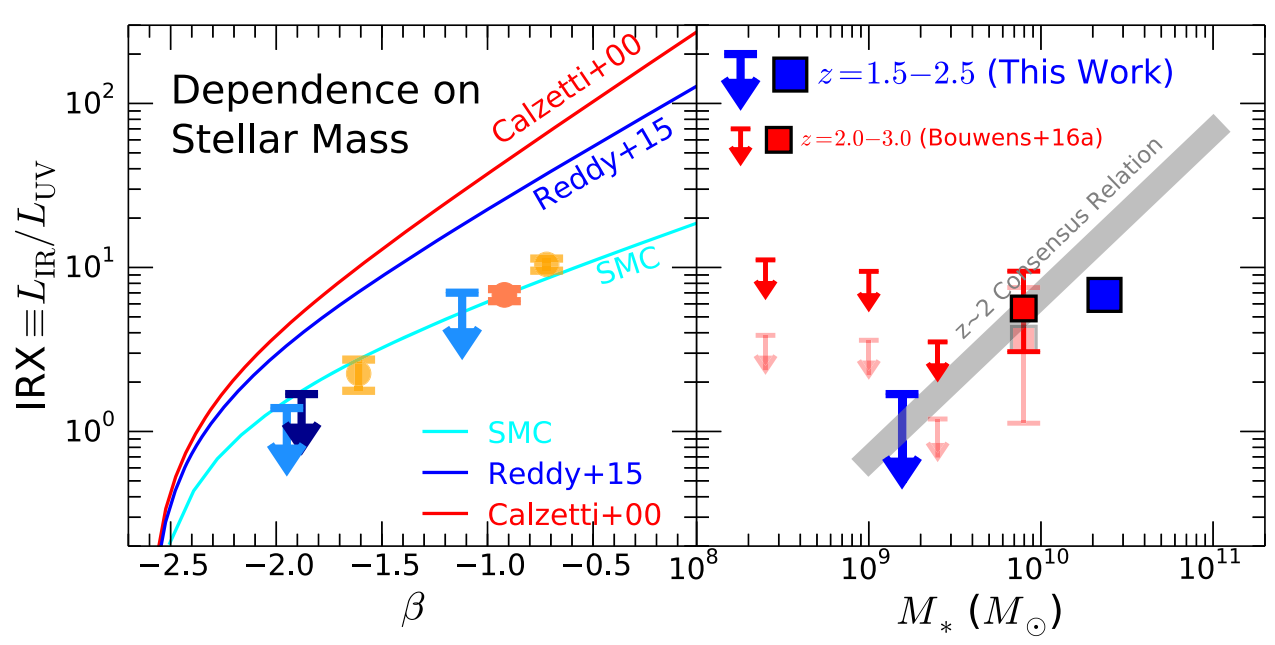

Figure 7. Left: IRX $-\beta$ relation as a function of stellar mass. The dark-blue arrow indicates the $3 \sigma$ upper limit for low-mass galaxies with $M_{*} \leqslant 10^{9.75} M_{\odot}$, while the light-blue arrows are for the low-mass subsample with $\beta \leqslant-1.4$ and $\beta>-1.4$. Similarly, the dark red and orange circles indicate the same for the high-mass $\left(M_{*}>10^{9.75} M_{\odot}\right)$ sample. Our measurements suggest that high-mass galaxies at $z=1.5-2.5$ are consistent with SMC extinction, while low-mass galaxies have upper limits in IRX that suggest a dust curve that is steeper than the SMC curve. Right: IRX as a function of stellar mass for galaxies in our sample (blue symbols) and that of Bouwens et al. (2016a) (red symbols), where the arrows indicate $3 \sigma$ upper limits. As the Bouwens et al. (2016a) measurements are based on ALMA 2 mm continuum data, the derived $L_{\mathrm{IR}}$ are sensitive to the assumed dust temperature. The dark- and light-red symbols indicate their results when assuming an evolving dust temperature and a constant temperature with $T=35 \mathrm{~K}$, respectively. The thick gray line denotes the "consensus relation" at $z \sim 2$ computed by Bouwens et al. (2016a) and based on data from Reddy et al. (2010), Whitaker et al. (2014), and Álvarez-Márquez et al. (2016).

counterparts (Reddy et al. 2008; Bouwens et al. 2009, 2012; Kurczynski et al. 2014). This inference is based on the fact that the observed UV luminosity is expected to monotonically correlate with SFR for galaxies fainter than $L^{*}$ (e.g., see Figure 13 of Reddy et al. 2010; Figure 10 of Bouwens et al. 2009) and that the dustiness is a strong function of SFR (Wang \& Heckman 1996; Adelberger \& Steidel 2000; Reddy et al. 2006a, 2010; Buat et al. 2007, 2009; Burgarella et al. 2009).

While several investigations have shown evidence for a correlation between IRX and UV luminosity (Bouwens et al. 2009; Reddy et al. 2010, 2012a), others point to a roughly constant IRX as a function of UV luminosity (Buat et al. 2009; Heinis et al. 2013). As discussed in these studies, the different findings may be a result of selection biases, in the sense that UV-selected samples will tend to miss the dustiest galaxies, which also have faint observed UV luminosities. Hence, for purely UV-selected samples, IRX would be expected to decrease with $L_{\mathrm{UV}}$. Alternatively, the rarity of highly dustobscured galaxies compared to intrinsically faint galaxies (e.g., as inferred from the shapes of the UV and IR luminosity functions; Caputi et al. 2007; Reddy et al. 2008; Reddy \& Steidel 2009; Magnelli et al. 2013) implies that in a numberweighted sense the mean bolometric luminosity should decrease toward fainter $L_{\mathrm{UV}}$. How this translates to the variation of IRX with $L_{\mathrm{UV}}$ will depend on how quickly the dust can build up in dynamically relaxed faint galaxies. From a physical standpoint, dust enrichment on timescales much shorter than the dynamical timescale would suggest a relatively constant IRX as a function of $L_{\mathrm{UV}}$.

The HDUV/3D-HST sample presents a unique opportunity to evaluate the trend between IRX and $L_{\mathrm{UV}}$, as the selection is based on rest-optical criteria. Consequently, our sample is less sensitive to the bias against dusty galaxies that are expected to be significant in UV-selected samples (e.g., Chapman et al. 2000; Barger et al. 2000; Buat et al. 2005; Burgarella et al. 2005; Reddy et al. 2005, 2008; Casey et al. 2014a). Indeed, Figure 1 shows that our sample includes a large number of UV-faint galaxies that are also quite dusty based on their red $\beta \gtrsim-1.4-$ these galaxies, while dusty, still have bolometric luminosities that are sufficiently faint to be undetected in the PACS imaging.

Figure 8 shows the relationship between dust attenuation and UV luminosity for our sample (gray points) and those of Heinis et al. (2013) at $z \sim 1.5$ and Álvarez-Márquez et al. (2016) at $z \sim 3$. The latter two indicate an IRX that is roughly constant with $L_{\mathrm{UV}}$, but one that is a factor of 2-3 offset toward higher IRX than found for our sample. This offset is easily explained by the fact that the IRX $-L_{\mathrm{UV}}$ relations for the $z \sim 1.5$ and $z \sim 3.0$ samples were determined from galaxies that are on average significantly redder than in our sample. In particular, most of the constraints on IRX $-L_{\mathrm{UV}}$ from these studies come from galaxies with $\beta \gtrsim-1.5$. When limiting our stacks to galaxies with $\beta>-1.4$ (red points in Figure 8), we find an excellent agreement with the IRX $-L_{\mathrm{UV}}$ relations found by Heinis et al. (2013) and Álvarez-Márquez et al. (2016). On the other hand, stacking those galaxies in our sample with $\beta \leqslant-1.4$ results in an IRX $-L_{\mathrm{UV}}$ relation that is not surprisingly offset toward lower IRX than for the sample as a whole.

Thus, while the IRX $-L_{\mathrm{UV}}$ relation appears to be roughly constant for all of the samples considered here, Figure 8 implies that the $\beta$ distribution as a function of $L_{\mathrm{UV}}$ is at least as important as the presence of dusty star-forming galaxies in shaping the observed IRX $-L_{\mathrm{UV}}$ relation. Furthermore, the trend of a bluer average $\beta$ with decreasing $L_{\mathrm{UV}}$ (e.g., Figure 1; see also Reddy et al. 2008; Bouwens et al. 2009) suggests that the mean reddening should be correspondingly lower for UVfaint galaxies than for UV-bright ones once the effect of the less numerous dusty galaxies with red $\beta$ is accounted for.

The blue $(\beta \leqslant-1.4)$ star-forming galaxies in our sample have IRX $\lesssim 1$, such that the infrared and UV luminosities contribute equally to the bolometric luminosities of these galaxies. The expectation of rapid dust enrichment from corecollapse supernovae (Todini \& Ferrara 2001) implies that it is unlikely that the dust obscuration can be significantly lower than this value when observed for dynamically relaxed 

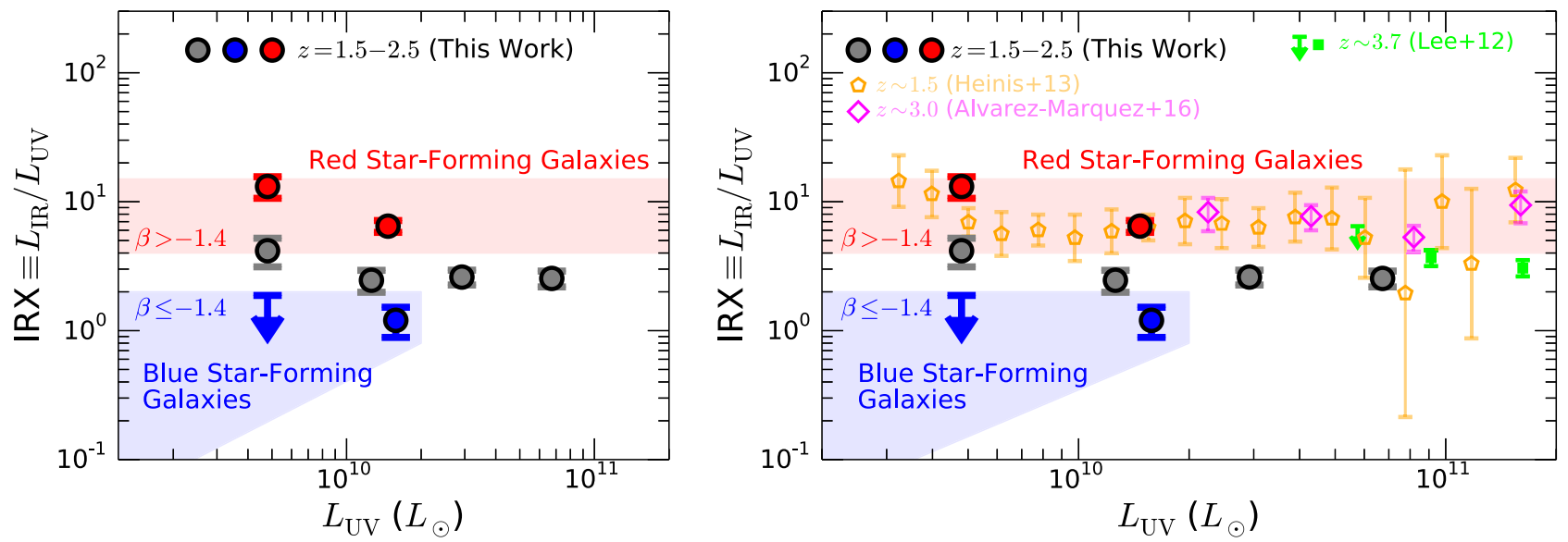

Figure 8. Dust attenuation as a function of UV luminosity at $z \sim 1.5-3.7$. Our stacked measurements in bins of $L_{\mathrm{UV}}$ are indicated by the large gray circles, and the large blue and red circles indicate the values obtained by further subdividing the stacks in bins of $\beta$ (see Table 2). The right panel also shows other results for UVselected galaxies at $z \sim 1.5$ (Heinis et al. 2013), $z \sim 3$ (Álvarez-Márquez et al. 2016), and $z \sim 3.7$ (Lee et al. 2012). The samples of Heinis et al. (2013) and ÁlvarezMárquez et al. (2016) include galaxies that are primarily redder $(\beta \gtrsim-1.4)$ than those in our sample. When restricting our sample to a similar range of $\beta$, we find an IRX $-L_{\mathrm{UV}}$ relation that is in excellent agreement with that of Heinis et al. (2013) and Álvarez-Márquez et al. (2016). Unsurprisingly, the IRX $-L_{\mathrm{UV}}$ relation for blue galaxies with $\beta \leqslant-1.4$ is offset by $\approx 1$ dex to lower IRX.

systems. Consequently, the observation that the mean UV slope becomes progressively bluer for fainter galaxies at high redshift (e.g., Bouwens et al. 2009; Wilkins et al. 2011; Finkelstein et al. 2012; Alavi et al. 2014) may simply be a result of systematic changes in metallicity and/or star formation history where the intrinsic UV slope also becomes bluer but IRX remains relatively constant (e.g., Figure 2; Wilkins et al. 2011, 2013; Alavi et al. 2014). Thus, the common observation that UV-faint galaxies are bluer than their brighter counterparts may not directly translate into a lower dust obscuration for the former.

Moreover, $\beta$ is relatively insensitive to IRX for $\beta-\beta_{0} \lesssim 0.2$ (e.g., Figure 2 ). Our results thus indicate caution when using the IRX $-\beta$ relation to infer the dust reddening of blue galaxies at high redshift, as such estimates may be highly dependent on the intrinsic UV slope of the stellar population and even otherwise quite uncertain if the difference in observed and intrinsic UV slopes is small.

\subsection{Young/Low-mass Galaxies}

ALMA has opened up new avenues for investigating the ISM and dust content of very high redshift galaxies, and a few recent efforts have focused in particular on the [C II] $158 \mu \mathrm{m}$ line in galaxies at $z \gtrsim 5$ (Capak et al. 2015; Maiolino et al. 2015; Schaerer et al. 2015; Watson et al. 2015; Willott et al. 2015; Knudsen et al. 2016; Pentericci et al. 2016) and dust continuum at far-IR and millimeter wavelengths (e.g., Fudamoto et al. 2017). Capak et al. (2015) report on ALMA constraints on the IRX of a small sample of $10 z \sim 5.5$ LBGs and find that they generally fall below the SMC extinction curve. The disparity between the SMC curve and their data points is increased if one adopts a bluer intrinsic slope than that assumed in Meurer et al. (1999), a reasonable expectation for these high-redshift and presumably lower-metallicity galaxies.

More generally, earlier results suggesting that "young" LBGs (ages $\lesssim 100 \mathrm{Myr}$ ) and/or those with lower stellar masses at $z \gtrsim 2$ are consistent with an SMC curve (Baker et al. 2001; Reddy et al. 2006b, 2012a; Siana et al. 2008, 2009; Reddy et al. 2010; Bouwens et al. 2016a) would also require a steeper-than-SMC curve if their intrinsic slopes are substantially bluer than that normally assumed in interpreting the IRX $-\beta$ relation. Unfortunately, there are only a very small number of galaxies in our sample that have SED-determined ages of $<100$ Myr (81 galaxies), and stacking them results in an unconstraining upper limit on IRX (Table 2).

Note that an ambiguity arises because the ages are derived from SED fitting, which assumes some form of the attenuation curve. Following Reddy et al. (2010), the number of galaxies considered "young" would be lower under the assumption of SMC extinction rather than Calzetti et al. (2000) attenuation, as the former results in lower $E(B-V)$ for a given UV slope, translating into older ages. Self-consistently modeling the SEDs based on the location of galaxies in the IRX $-\beta$ plane results in fewer $<100 \mathrm{Myr}$ galaxies, but of course their location in the IRX $-\beta$ plane is unaffected (Reddy et al. 2010), as is the conclusion that such young galaxies would require a dust curve steeper than that of the SMC if they have blue intrinsic UV slopes. In addition, as noted in Section 5.2, galaxies in our lowmass $\left(M_{*} \leqslant 10^{9.75} M_{\odot}\right)$ subsample appear to also require a dust curve steeper than that of the SMC.

Figure 9 summarizes a few recent measurements of IRX $-\beta$ for young and low-mass galaxies at $z \sim 2$ (Baker et al. 2001; Siana et al. 2009; Reddy et al. 2010, 2012a) and low-mass galaxies and LBGs in general at $z \sim 4-10$ (Bouwens et al. 2016a), as well as our own measurements. The compilation from Reddy et al. (2010, 2012a) includes $24 \mu \mathrm{m}$ constraints on the IRX of young galaxies. Shifting their IRX by $\approx 0.35$ dex to higher values to account for the deficit of PAH emission in galaxies with $\lesssim 400$ Myr (Shivaei et al. 2017) results in upper limits or a stacked measurement of IRX that are broadly consistent with either the SMC curve or one that is steeper.

Considering the Herschel measurements here and in Reddy et al. (2012a) and ALMA measurements at $z \sim 2-10$ (Capak et al. 2015; Bouwens et al. 2016a), we find that young/lowmass galaxies at $z \gtrsim 2$ follow a dust curve steeper than that of the SMC, particularly in the context of a blue intrinsic slope, $\beta_{0}=-2.62$. Note that unlike cB58, which has a stellar metallicity of $\simeq 0.25 Z_{\odot}$ (Pettini et al. 2000), the Cosmic Eye has a metallicity of $\sim 0.5 Z_{\odot}$, suggesting a relatively red intrinsic UV slope. In this case, the IRX of the Cosmic Eye may be described adequately by the SMC curve. There is 


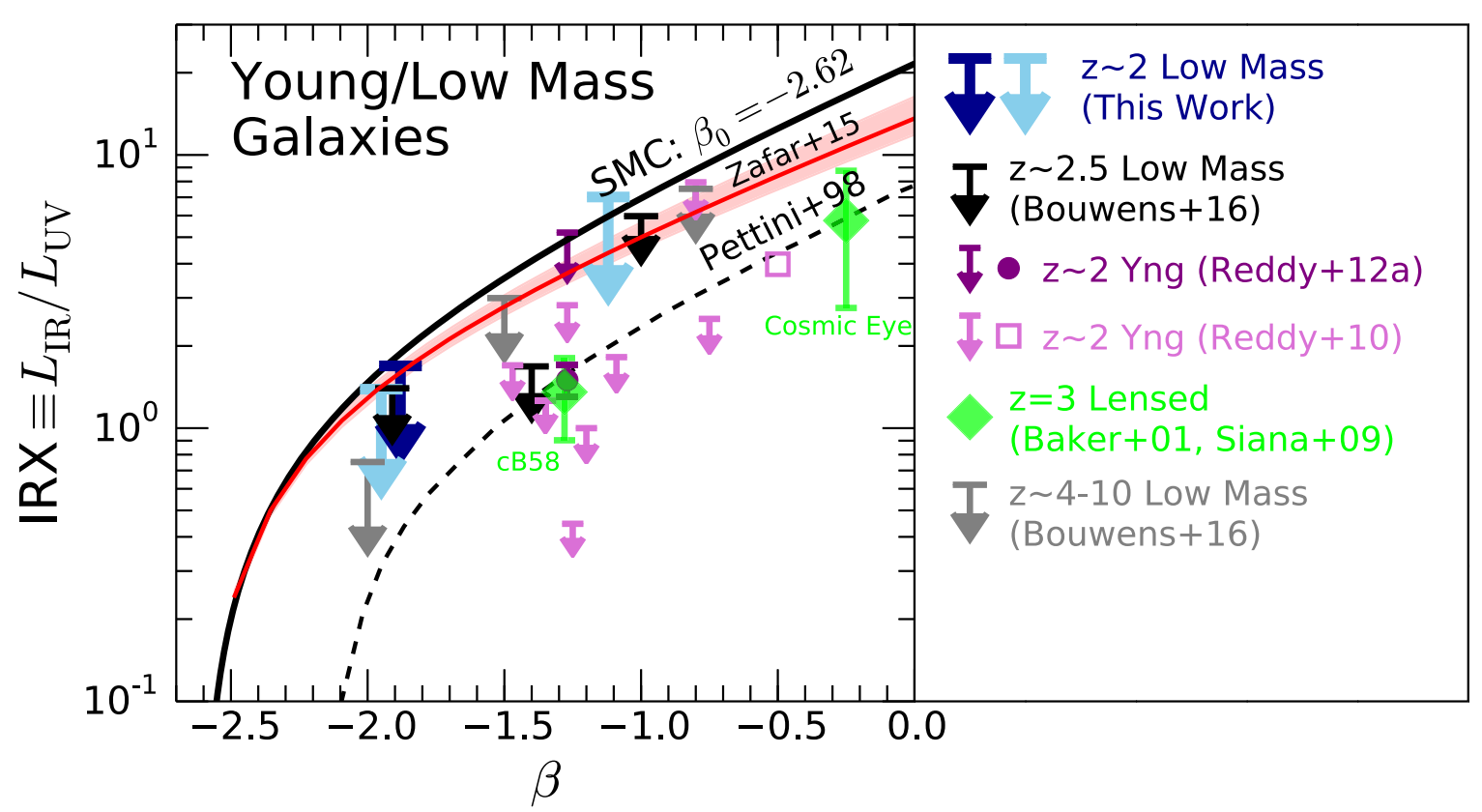

Figure 9. IRX $-\beta$ diagram for young and/or low-mass galaxies at $z \gtrsim 2$. The upper limits in IRX for low-mass $\left(M_{*} \leqslant 10^{9.75} M_{\odot}\right)$ galaxies in our sample are indicated by the dark- and light-blue arrows, the same as those shown in the left panel of Figure 7. Upper limits in IRX derived from ALMA measurements for low-mass $\left(M_{*} \leqslant 10^{9.75} M_{\odot}\right)$ galaxies at $z \sim 2-3$ are indicated by the dark-gray arrows (Bouwens et al. 2016a). The upper limit in IRX derived from a Herschel/PACS stack for UV-selected $z \sim 2$ galaxies with ages $\lesssim 100 \mathrm{Myr}$ is indicated by the purple arrow, while the purple filled circle indicates the stacked inference from Spitzer/MIPS $24 \mu \mathrm{m}$ data (Reddy et al. 2012a). Similarly, the light-purple arrows and open square denote the upper limits for individual $<100$ Myr galaxies and the stacked result, respectively, derived from $24 \mu \mathrm{m}$ data (Reddy et al. 2010). Also shown are results from two lensed galaxies (large green diamonds; cB58 and the Cosmic Eye; Baker et al. 2001; Siana et al. 2009) and upper limits for low-mass galaxies at $z \sim 4-10$ (light-gray arrows; Bouwens et al. 2016a). For reference, the black dashed line shows the SMC curve as parameterized by Pettini et al. (1998), while the red line and shaded region denote the average and dispersion in the IRX- $\beta$ relation for the average extinction curve derived for quasars (Zafar et al. 2015). For clarity, the results from Capak et al. (2015) for $z \sim 5.5$ LBGs are not shown, but we note that most of their points lie at least $1 \sigma$ below the Pettini et al. (1998) curve shown in the figure. The detections and upper limits for young and low-mass galaxies at a variety of redshifts suggest a dust curve that is on average steeper than the SMC curve, particularly if we assume a blue intrinsic UV slope of $\beta_{0}=-2.62$ (thick black solid line).

additional evidence of suppressed IRX ratios at lower mass from rest-optical emission line studies of $z \sim 2$ galaxies. In particular, Reddy et al. (2015) found that a significant fraction of $z \sim 2$ galaxies with $M_{*} \lesssim 10^{9.75} M_{\odot}$ have very red $\beta$, or $E$ $(B-V)$, relative to the reddening deduced from the Balmer lines (e.g., see their Figure 17), implying that such galaxies would have lower IRX for a given $\beta$ than that predicted by common attenuation/extinction curves.

Evidence for curves steeper than the SMC average has been observed along certain sightlines within the SMC (Gordon et al. 2003), in the Milky Way and some Local Group galaxies (Gordon et al. 2003, 2009; Sofia et al. 2005; Amanullah et al. 2014), and in quasars (e.g., Hall et al. 2002; Jiang et al. 2013; Zafar et al. 2015). Our results, combined with those in the literature, suggest that such steep curves may be typical of low-mass and young galaxies at high redshift. While the attenuation curve will undoubtedly vary from galaxy to galaxy depending on the star formation history, age, metallicity, dust composition, and geometrical configuration of stars and dust, the fact that young/low-mass galaxies lie systematically below the IRX $-\beta$ relation predicted with an SMC curve suggests that a steep curve may apply uniformly to such galaxies.

An unresolved issue is the physical reason why young and low-mass galaxies may follow a steeper attenuation curve than their older and more massive counterparts. Reddy et al. (2012a) suggest a possible scenario in which galaxies transition from steep to gray attenuation curves as they age owing to star formation occurring over extended regions and/or the cumulative effects of galactic outflows that are able to clear the gas and dust along certain sightlines. On the other hand, if young and low-mass galaxies have higher ionizing escape fractions as a result of lower gas/ dust covering fractions (e.g., Reddy et al. 2016b), then one might expect their attenuation curve to exhibit a shallower dependence on wavelength than the SMC extinction curve.

In any case, curves steeper than the SMC may be possible with a paucity of large dust grains and/or an overabundance of silicate grains (Zafar et al. 2015). In particular, large dust grains may be efficiently destroyed by $\mathrm{SN}$ shock waves (Draine \& Salpeter 1979; McKee 1989; Jones 2004), which would have the effect of steepening the dust curve (i.e., such that proportionally more light is absorbed in the UV relative to the optical). If the destruction of large grains is significant in young/low-mass galaxies, then it may explain both their red $\beta$ and their low IRX. Alternatively, the lower gas-phase [Si/O] measured from the composite rest-frame UV spectrum of $z \sim 2$ galaxies relative to the solar value indicates significant depletion of Si onto dust grains, while carbon is underabundant relative to oxygen (Steidel et al. 2016). This result may suggest an enhancement of silicate over carbonaceous grains that results in a steeper attenuation curve. Finally, the positions of very high redshift galaxies in the IRX $-\beta$ plane, particularly those with with lower IRX than what would be predicted with an SMC-like curve, may be dictated more by geometrical considerations (e.g., Reddy et al. 2006b; Faisst et al. 2017) than by a physical change in the dust grain population. 

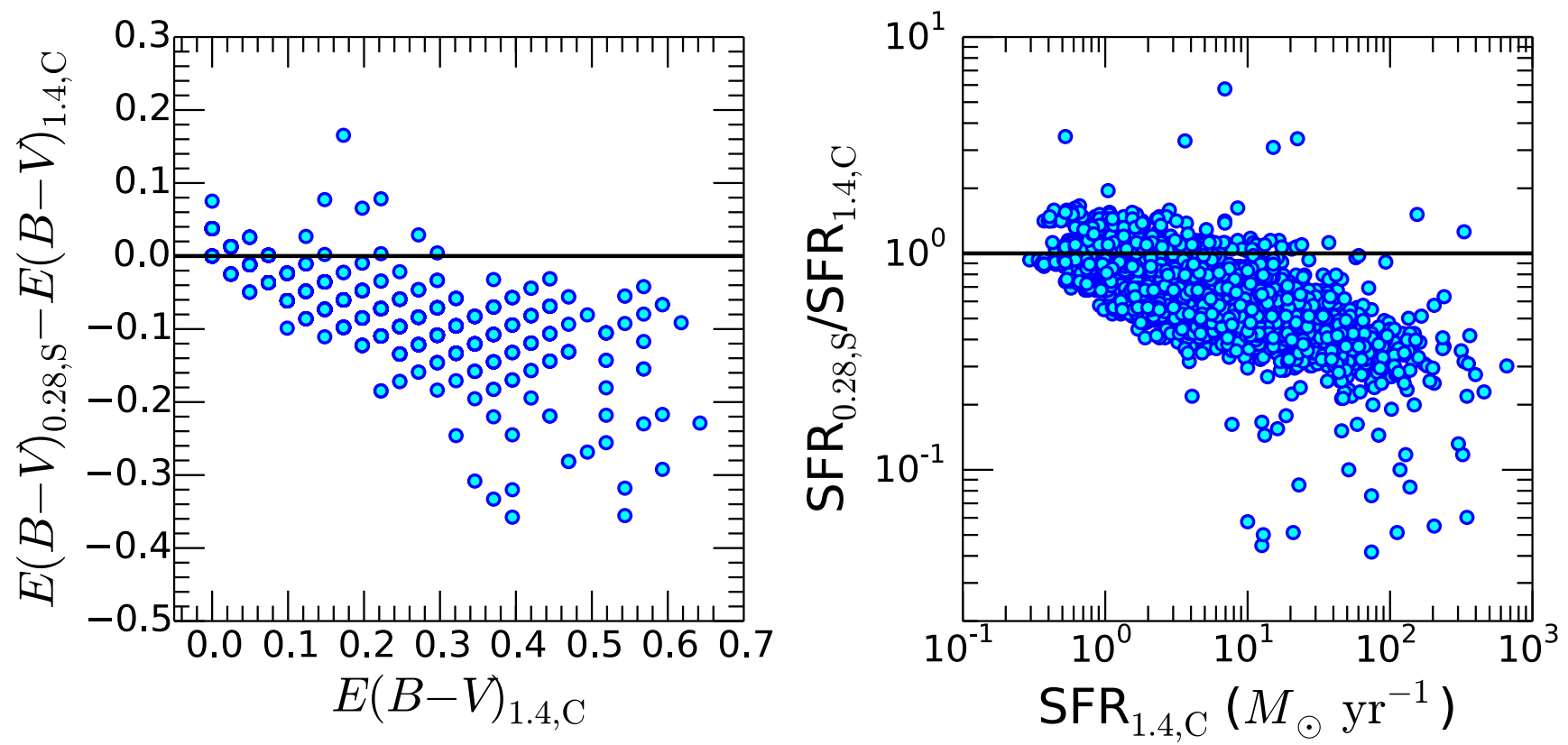

Figure 10. Comparison of the derived reddening, $E(B-V)$, and SFRs when considering the following cases: (a) $1.4 Z_{\odot}$ BC03 model with the Calzetti et al. (2000) attenuation curve ("1.4,C" subscripts), and (b) $0.28 Z_{\odot}$ BC03 model with SMC extinction curve (" $0.28, S$ ") subscripts. The typical random uncertainties are $\delta E(B-V) \simeq 0.04$ and $\delta \log \left[\mathrm{SFR} / M_{\odot} \mathrm{yr}^{-1}\right] \simeq 0.17$ dex. The sparse sampling in the left panel is due to the fixed increments of $\delta A_{\mathrm{V}}=0.1$ used when fitting the SEDs of galaxies in our sample. The difference in reddening between the two cases becomes increasingly discrepant for objects with redder colors. A lower $E(B-V)$ is required to reproduce an observed UV slope given the steep far-UV rise of the SMC extinction curve compared to that of the Calzetti et al. (2000) curve. These differences in reddening, combined with the particular values of the SMC and Calzetti et al. (2000) attenuation curves at $\lambda=1600 \AA$, result in SFRs that are generally lower in case (b) than in case (a).

\subsection{Implications for Stellar Populations and Ionizing Production Efficiencies}

Inferring the intrinsic stellar populations of galaxies based on their observed photometry requires one to adopt some form of the dust attenuation curve. It is therefore natural to ask whether these inferences change in light of our findings of a steeper (SMC-like) attenuation curve for $z \sim 2$ galaxies with intrinsically blue UV spectral slopes. To address this issue, we remodeled (using FAST) the SEDs of galaxies in our sample assuming two cases: (a) a $1.4 Z_{\odot}$ BC03 model (canonically referred to as the "solar"-metallicity model using old solar abundance measurements) with the Calzetti et al. (2000) attenuation curve, and (b) a $0.28 Z_{\odot}$ BC03 model with an SMC extinction curve. The ages derived in case (b) are on average $30 \%$ older than those derived in case (a), primarily because less reddening is required to reproduce a given UV slope with the SMC extinction curve, resulting in older ages (e.g., see discussion in Reddy et al. 2012b). Similarly, the stellar masses derived in case (b) are on average 30\% lower than those derived in case (a).

Perhaps most relevant in the context of our analysis are the changes in inferred reddening and SFR. As shown in Figure 10, the reddening deduced from the SMC extinction curve is lower than that obtained with the Calzetti et al. (2000) attenuation curve, owing to the steep rise in the far-UV of the former relative to the latter. The largest differences in $E(B-V)$ and SFR occur for the reddest objects and those with larger SFRs. We find the following relations between the reddening and SFRs derived for the two cases discussed above:

$$
E(B-V)_{0.28 Z_{\odot}, \mathrm{SMC}}=0.65 E(B-V)_{1.4 Z_{\odot}, \text { Calz }}
$$

and

$$
\begin{aligned}
& \log \left[\mathrm{SFR}_{0.28 Z_{\odot}, \mathrm{SMC}} / M_{\odot} \mathrm{yr}^{-1}\right] \\
& \quad=0.79 \log \left[\mathrm{SFR}_{1.4 \mathrm{Z}_{\odot}, \mathrm{Calz}} / M_{\odot} \mathrm{yr}^{-1}\right]-0.05 .
\end{aligned}
$$

The lower SFRs derived in the SMC case result in a factor of $\approx 2$ lower SFR densities at $z \gtrsim 2$, as discussed in some depth in Bouwens et al. (2016a).

The general applicability of an SMC-like dust curve to highredshift galaxies is also of particular interest when considering its impact on the ionizing efficiencies of such galaxies, a key input to reionization models (Robertson et al. 2013; Bouwens et al. 2015, 2016b). Specifically, the ionizing photon production efficiency, $\xi_{\text {ion }}$, is simply the ratio of the rate of H-ionizing photons produced to the nonionizing UV continuum luminosity. This quantity is directly related to another commonly used ratio, namely, the Lyman continuum flux density (e.g., at 800 or $900 \AA$ ) to the nonionizing UV flux density (e.g., at $1600 \AA$ ), $f_{900} / f_{1600}$.

The ionizing photon production efficiency is typically computed by combining Balmer emission lines, such as $\mathrm{H} \alpha$, with UV continuum measurements (e.g., Bouwens et al. 2015), but both the lines and the continuum must be corrected for dust attenuation. In the context of our present analysis, the dust corrections for the UV continuum are lower by factors of 1.12, 1.82, and 2.95 for galaxies with Calzetti-inferred SFRs of 1,10 , and $100 M_{\odot} \mathrm{yr}^{-1}$, respectively. Thus, for given Balmer line luminosities that are corrected for dust using the Galactic extinction curve (Calzetti et al. 1994; Steidel et al. 2014; Reddy et al. 2015), $\xi_{\text {ion }}$ would be correspondingly larger by the same factors by which the dust-corrected UV luminosities are lower. 
A secondary effect that will boost $\xi_{\text {ion }}$ above the predictions from single-star solar-metallicity stellar population models is the higher ionizing photon output associated with lower-metallicity and rotating massive stars (Eldridge \& Stanway 2009; Leitherer et al. 2014). In particular, the fiducial $0.14 Z_{\odot}$ BPASS model that includes binary evolution and an IMF extending to $300 \mathrm{M}_{\odot}$ predicts a factor of $\approx 3$ larger $\mathrm{H} \alpha$ luminosity per solar mass of star formation after $100 \mathrm{Myr}$ of constant star formation relative to that computed using the Kennicutt \& Evans (2012) relation, which assumes a solar-metallicity Starburst 99 model. On the other hand, the UV luminosity is larger by $\approx 30 \%$. Thus, such models predict a $\xi_{\text {ion }}$ that is elevated by a factor of $\approx 2$ relative to those assumed in standard calibrations between $\mathrm{H} \alpha / \mathrm{UV}$ luminosity and SFR (see, e.g., Stark et al. 2015; Bouwens et al. 2016b; Nakajima et al. 2016; Reddy et al. 2016b). Consequently, calculations or predictions of $\xi_{\text {ion }}$ for high-redshift galaxies should take into account the effects of a steeper attenuation curve and lower-metallicity stellar populations that may include stellar rotation/binarity. Our results suggest that an elevated value of $\xi_{\text {ion }}$ not only is a feature of very high redshift $(z \gtrsim 6)$ galaxies, but also may be quite typical for $z \sim 2$ galaxies.

\section{Conclusions}

In this paper, we have presented an analysis of the relationship between dust obscuration (IRX $\left.=L_{\mathrm{IR}} / L_{\mathrm{UV}}\right)$ and other commonly derived galaxy properties, including UV slope $(\beta)$, stellar mass $\left(M_{*}\right)$, and UV luminosity $\left(L_{\mathrm{UV}}\right)$, for a large sample of 3545 rest-optical-selected star-forming galaxies at $z=1.5-2.5$ drawn from HDUV UVIS and 3D-HST photometric catalogs of the GOODS fields. Our sample is unique in that it significantly extends the dynamic range in $\beta$ and $L_{\mathrm{UV}}$ compared to previous UV-selected samples at these redshifts. In particular, close to $60 \%$ of the objects in our sample have UV slopes bluer than $\beta=-1.70$, and $>95 \%$ have rest-frame UV absolute magnitudes fainter than the characteristic magnitude at these redshifts, with the faintest galaxies having $L_{\mathrm{UV}} \approx 0.05 L_{\mathrm{UV}}^{*}$.

We use stacks of the deep Herschel/PACS imaging in the GOODS fields to measure the average dust obscuration for galaxies in our sample and compare it to predictions of the IRX $-\beta$ relation for different stellar population models and attenuation/extinction curves using energy balance arguments. Specifically, we consider the commonly adopted Bruzual \& Charlot (2003) stellar population models for different metallicities $\left(0.28\right.$ and $\left.1.4 Z_{\odot}\right)$, as well as the low-metallicity $\left(0.14 Z_{\odot}\right)$ BPASS model. Additionally, we compute predictions of the IRX $-\beta$ relations for the Calzetti et al. (2000) and Reddy et al. (2015) dust attenuation curves and the SMC extinction curve. The lower-metallicity stellar population models result in significant shifts in the IRX $-\beta$ relation of up to $\delta \beta=0.4$ toward bluer $\beta$ relative to the canonical relation of Meurer et al. (1999). In the context of the lower-metallicity stellar population models applicable for high-redshift galaxies, we find that the strong trend between IRX and $\beta$ measured from the HDUV and 3D-HST samples follows most closely that predicted by the SMC extinction curve. We find that grayer attenuation curves (e.g., Calzetti et al. 2000) overpredict the IRX at a given $\beta$ by a factor of $\gtrsim 3$ when assuming intrinsically blue UV spectral slopes. Thus, our results suggest that an SMC curve is the one most applicable to lower stellar metallicity populations at high redshift.

Performing a complementary stacking analysis of the Spitzer/MIPS $24 \mu \mathrm{m}$ images implies an average mid-IR-to-IR luminosity ratio, $\left\langle L_{7.7} / L_{\mathrm{IR}}\right\rangle$, that is a factor of 3-4 lower than for galaxies with reddest $\beta(>-0.5)$ and the UV-brightest $\left(M_{1600} \lesssim-21\right)$ and UV-faintest $\left(M_{1600} \gtrsim-19\right)$ galaxies relative to the average for all galaxies in our sample (Appendix B). These results indicate large variations in the conversion between rest-frame $7.7 \mu \mathrm{m}$ and IR luminosity.

At any given UV luminosity, galaxies with redder $\beta$ have larger IRX. IRX $-L_{\mathrm{UV}}$ relations for blue and red star-forming galaxies average together to result in a roughly constant IRX of $\simeq 3-4$ over roughly two decades in UV luminosity $\left(2 \times 10^{9}\right.$ $\left.L_{\odot} \lesssim L_{\mathrm{UV}} \lesssim 2 \times 10^{11} L_{\odot}\right)$. Consequently, the bluer $\beta$ observed for UV-faint galaxies seen in this work and previous studies may simply reflect intrinsically bluer UV spectral slopes for such galaxies, rather than signifying changes in the dust obscuration.

Galaxies with stellar masses $M_{*}>10^{9.75} M_{\odot}$ have an IRX $-\beta$ relation that is consistent with the SMC extinction curve, while the lower-mass galaxies in our sample with $M_{*} \leqslant 10^{9.75} M_{\odot}$ have an IRX $-\beta$ relation that is at least as steep as the SMC. The shifting of the IRX $-\beta$ relations toward bluer $\beta$ for the lowermetallicity stellar populations expected for high-redshift galaxies implies that the low-mass galaxies in our sample, as well as the low-mass and young galaxies from previous studies, require a dust curve steeper than that of the SMC. The low-metallicity stellar populations favored for high-redshift galaxies result in steeper attenuation curves and higher ionizing photon production rates, which, in turn, facilitate the role that galaxies may have in reionizing the universe at very high redshift or keeping the universe ionized at lower redshifts $(z \sim 2)$.

There are several future avenues for building on this work. Detailed spectral modeling of the rest-UV and/or rest-optical spectra of galaxies (e.g., Reddy et al. 2016b; Steidel et al. 2016) may be used to discern their intrinsic spectral slopes and thus disentangle the effects of the intrinsic $\beta$ and the attenuation curve relevant for high-redshift galaxies. Second, the higher spatial resolution and depth of X-ray observations (compared to the far-IR) make them advantageous for investigating the bolometric SFRs and hence dust obscuration for galaxies substantially fainter than those directly detected with either Spitzer or Herschel in reasonable amounts of time, provided that the scaling between X-ray luminosity and SFR can be properly calibrated (e.g., for metallicity effects; Basu-Zych et al. 2013). In addition, nebular recombination line estimates of dust attenuation (e.g., from the Balmer decrement; Reddy et al. 2015) may be used to assess the relationship between IRX and $\beta$ for individual star-forming galaxies, rather than through the stacks necessitated by the limited sensitivity of far-IR imaging.

This work was supported by NASA through grant HST-GO13871 and from the Space Telescope Science Institute, which is operated by AURA, Inc., under NASA contract NAS 5-26555. $\mathrm{K}$. Penner kindly provided data from his published work in electronic format. N.A.R. is supported by an Alfred P. Sloan Research Fellowship. 
Table 3

Summary of $\beta$ vs. $E(B-V)$ and IRX versus $\beta$ Relations

\begin{tabular}{|c|c|c|c|c|c|c|}
\hline Model $^{\mathrm{a}}$ & Dust Curve $^{\mathrm{b}}$ & $\begin{array}{c}\text { Nebular } \\
\text { Continuum }^{\mathrm{c}}\end{array}$ & $\begin{array}{c}\text { Ly } \alpha \\
\text { Heating }\end{array}$ & $\delta R_{\mathrm{V}}{ }^{\mathrm{e}}$ & $\beta^{\mathrm{f}}$ & IRX \\
\hline \multirow[t]{24}{*}{ BPASS $-0.14 Z_{\odot}$} & \multirow[t]{8}{*}{ Calzetti+00 } & Yes & Yes & 0.0 & \multirow[t]{4}{*}{$-2.616+4.684 E(B-V)$} & $1.67 \times\left[10^{0.4(2.13 \beta+5.57)}-1\right]$ \\
\hline & & Yes & Yes & 1.5 & & $1.66 \times\left[10^{0.4(1.81 \beta+4.73)}-1\right]$ \\
\hline & & Yes & No & 0.0 & & $1.39 \times\left[10^{0.4(2.13 \beta+5.57)}-1\right]$ \\
\hline & & Yes & No & 1.5 & & $1.38 \times\left[10^{0.4(1.81 \beta+4.73)}-1\right]$ \\
\hline & & No & Yes & 0.0 & \multirow[t]{4}{*}{$-2.709+4.684 E(B-V)$} & $1.73 \times\left[10^{0.4(2.13 \beta+5.76)}-1\right]$ \\
\hline & & No & Yes & 1.5 & & $1.73 \times\left[10^{0.4(1.81 \beta+4.90)}-1\right]$ \\
\hline & & No & No & 0.0 & & $1.44 \times\left[10^{0.4(2.13 \beta+5.76)}-1\right]$ \\
\hline & & No & No & 1.5 & & $1.42 \times\left[10^{0.4(1.81 \beta+4.90)}-1\right]$ \\
\hline & \multirow[t]{8}{*}{ Reddy +15} & Yes & Yes & 0.0 & \multirow[t]{4}{*}{$-2.616+4.594 E(B-V)$} & $1.68 \times\left[10^{0.4(1.82 \beta+4.77)}-1\right]$ \\
\hline & & Yes & Yes & 1.5 & & $1.67 \times\left[10^{0.4(1.50 \beta+3.92)}-1\right]$ \\
\hline & & Yes & No & 0.0 & & $1.40 \times\left[10^{0.4(1.82 \beta+4.77)}-1\right]$ \\
\hline & & Yes & No & 1.5 & & $1.38 \times\left[10^{0.4(1.50 \beta+3.92)}-1\right]$ \\
\hline & & No & Yes & 0.0 & \multirow[t]{4}{*}{$-2.709+4.594 E(B-V)$} & $1.74 \times\left[10^{0.4(1.82 \beta+4.94)}-1\right]$ \\
\hline & & No & Yes & 1.5 & & $1.74 \times\left[10^{0.4(1.50 \beta+4.05)}-1\right]$ \\
\hline & & No & No & 0.0 & & $1.44 \times\left[10^{0.4(1.82 \beta+4.94)}-1\right]$ \\
\hline & & No & No & 1.5 & & $1.43 \times\left[10^{0.4(1.50 \beta+4.05)}-1\right]$ \\
\hline & \multirow[t]{8}{*}{ SMC (Gordon+03) } & Yes & Yes & 0.0 & \multirow[t]{4}{*}{$-2.616+11.259 E(B-V)$} & $1.79 \times\left[10^{0.4(1.07 \beta+2.79)}-1\right]$ \\
\hline & & Yes & Yes & 1.5 & & $1.80 \times\left[10^{0.4(0.93 \beta+2.44)}-1\right]$ \\
\hline & & Yes & No & 0.0 & & $1.47 \times\left[10^{0.4(1.07 \beta+2.79)}-1\right]$ \\
\hline & & Yes & No & 1.5 & & $1.47 \times\left[10^{0.4(0.93 \beta+2.44)}-1\right]$ \\
\hline & & No & Yes & 0.0 & \multirow[t]{4}{*}{$-2.709+11.259 E(B-V)$} & $1.83 \times\left[10^{0.4(1.07 \beta+2.89)}-1\right]$ \\
\hline & & No & Yes & 1.5 & & $1.85 \times\left[10^{0.4(0.93 \beta+2.52)}-1\right]$ \\
\hline & & No & No & 0.0 & & $1.50 \times\left[10^{0.4(1.07 \beta+2.89)}-1\right]$ \\
\hline & & No & No & 1.5 & & $1.50 \times\left[10^{0.4(0.93 \beta+2.52)}-1\right]$ \\
\hline \multirow[t]{24}{*}{$\mathrm{BC} 03-1.4 Z_{\odot}$} & \multirow[t]{8}{*}{ Calzetti+00 } & Yes & Yes & 0.0 & \multirow[t]{4}{*}{$-2.383+4.661 E(B-V)$} & $1.44 \times\left[10^{0.4(2.14 \beta+5.10)}-1\right]$ \\
\hline & & Yes & Yes & 1.5 & & $1.41 \times\left[10^{0.4(1.82 \beta+4.33)}-1\right]$ \\
\hline & & Yes & No & 0.0 & & $1.35 \times\left[10^{0.4(2.14 \beta+5.10)}-1\right]$ \\
\hline & & Yes & No & 1.5 & & $1.32 \times\left[10^{0.4(1.82 \beta+4.33)}-1\right]$ \\
\hline & & No & Yes & 0.0 & \multirow[t]{4}{*}{$-2.439+4.661 E(B-V)$} & $1.47 \times\left[10^{0.4(2.14 \beta+5.22)}-1\right]$ \\
\hline & & No & Yes & 1.5 & & $1.44 \times\left[10^{0.4(1.82 \beta+4.43)}-1\right]$ \\
\hline & & No & No & 0.0 & & $1.38 \times\left[10^{0.4(2.14 \beta+5.22)}-1\right]$ \\
\hline & & No & No & 1.5 & & $1.35 \times\left[10^{0.4(1.82 \beta+4.43)}-1\right]$ \\
\hline & \multirow[t]{8}{*}{ Reddy +15} & Yes & Yes & 0.0 & \multirow[t]{4}{*}{$-2.383+4.568 E(B-V)$} & $1.43 \times\left[10^{0.4(1.83 \beta+4.37)}-1\right]$ \\
\hline & & Yes & Yes & 1.5 & & $1.39 \times\left[10^{0.4(1.51 \beta+3.59)}-1\right]$ \\
\hline & & Yes & No & 0.0 & & $1.40 \times\left[10^{0.4(1.82 \beta+4.77)}-1\right]$ \\
\hline & & Yes & No & 1.5 & & $1.30 \times\left[10^{0.4(1.51 \beta+3.59)}-1\right]$ \\
\hline & & No & Yes & 0.0 & \multirow[t]{4}{*}{$-2.439+4.568 E(B-V)$} & $1.46 \times\left[10^{0.4(1.83 \beta+4.47)}-1\right]$ \\
\hline & & No & Yes & 1.5 & & $1.42 \times\left[10^{0.4(1.51 \beta+3.67)}-1\right]$ \\
\hline & & No & No & 0.0 & & $1.37 \times\left[10^{0.4(1.83 \beta+4.47)}-1\right]$ \\
\hline & & No & No & 1.5 & & $1.33 \times\left[10^{0.4(1.51 \beta+3.67)}-1\right]$ \\
\hline & \multirow[t]{8}{*}{ SMC (Gordon+03) } & Yes & Yes & 0.0 & \multirow[t]{4}{*}{$-2.383+11.192 E(B-V)$} & $1.47 \times\left[10^{0.4(1.07 \beta+2.55)}-1\right]$ \\
\hline & & Yes & Yes & 1.5 & & $1.45 \times\left[10^{0.4(0.94 \beta+2.23)}-1\right]$ \\
\hline & & Yes & No & 0.0 & & $1.38 \times\left[10^{0.4(1.07 \beta+2.55)}-1\right]$ \\
\hline & & Yes & No & 1.5 & & $1.35 \times\left[10^{0.4(0.94 \beta+2.23)}-1\right]$ \\
\hline & & No & Yes & 0.0 & \multirow[t]{4}{*}{$-2.439+11.192 E(B-V)$} & $1.50 \times\left[10^{0.4(1.07 \beta+2.61)}-1\right]$ \\
\hline & & No & Yes & 1.5 & & $1.49 \times\left[10^{0.4(0.94 \beta+2.29)}-1\right]$ \\
\hline & & No & No & 0.0 & & $1.40 \times\left[10^{0.4(1.07 \beta+2.61)}-1\right]$ \\
\hline & & No & No & 1.5 & & $1.38 \times\left[10^{0.4(0.94 \beta+2.29)}-1\right]$ \\
\hline Meurer+99 & $\ldots$ & $\ldots$ & $\ldots$ & $\ldots$ & $-2.23+5.01 E(B-V)$ & $2.07 \times\left[10^{0.4(1.99 \beta+4.43)}-1\right]$ \\
\hline
\end{tabular}

Notes.

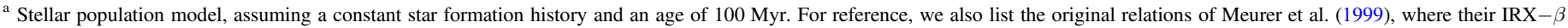
relation is shifted to higher IRX by 0.24 dex (see text).

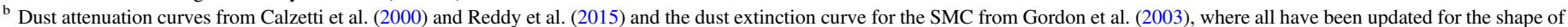
the curve at $\lambda \lesssim 1200 \AA$ according to the procedure of Reddy et al. (2016a).

${ }^{c}$ Indicates whether the nebular continuum emission is included.

${ }^{\mathrm{d}}$ Indicates whether dust heating by Ly $\alpha$ photons is included.

${ }^{\mathrm{e}}$ Indicates the value by which the dust attenuation curves are shifted downward in normalization $\left(R_{\mathrm{V}}\right)$.

${ }^{\mathrm{f}} \mathrm{A}$ blank entry indicates that the relation between $\beta$ and $E(B-V)$ is the same as that of the previous nonblank entry in this column.

\section{Appendix A $E(B-V)$ versus $\beta$ and IRX versus $\beta$ Relations}

In Table 3, we summarize the relations between $\beta$ and $E$ $(B-V)$ and between IRX and $\beta$ for different assumptions of the dust curve, heating from $\mathrm{Ly} \alpha$, inclusion of nebular continuum emission, and the normalization of the dust curve.

\section{Appendix B} Inferences of IRX from Spitzer/MIPS $24 \boldsymbol{\mu m}$ Data

Prior to the launch of Herschel, most non-UV-based inferences of the dust content of $L^{*}$ galaxies at $z \gtrsim 1.5$ relied on the detection of the redshifted mid-IR emission bands, commonly associated with PAHs, with the Spitzer MIPS 


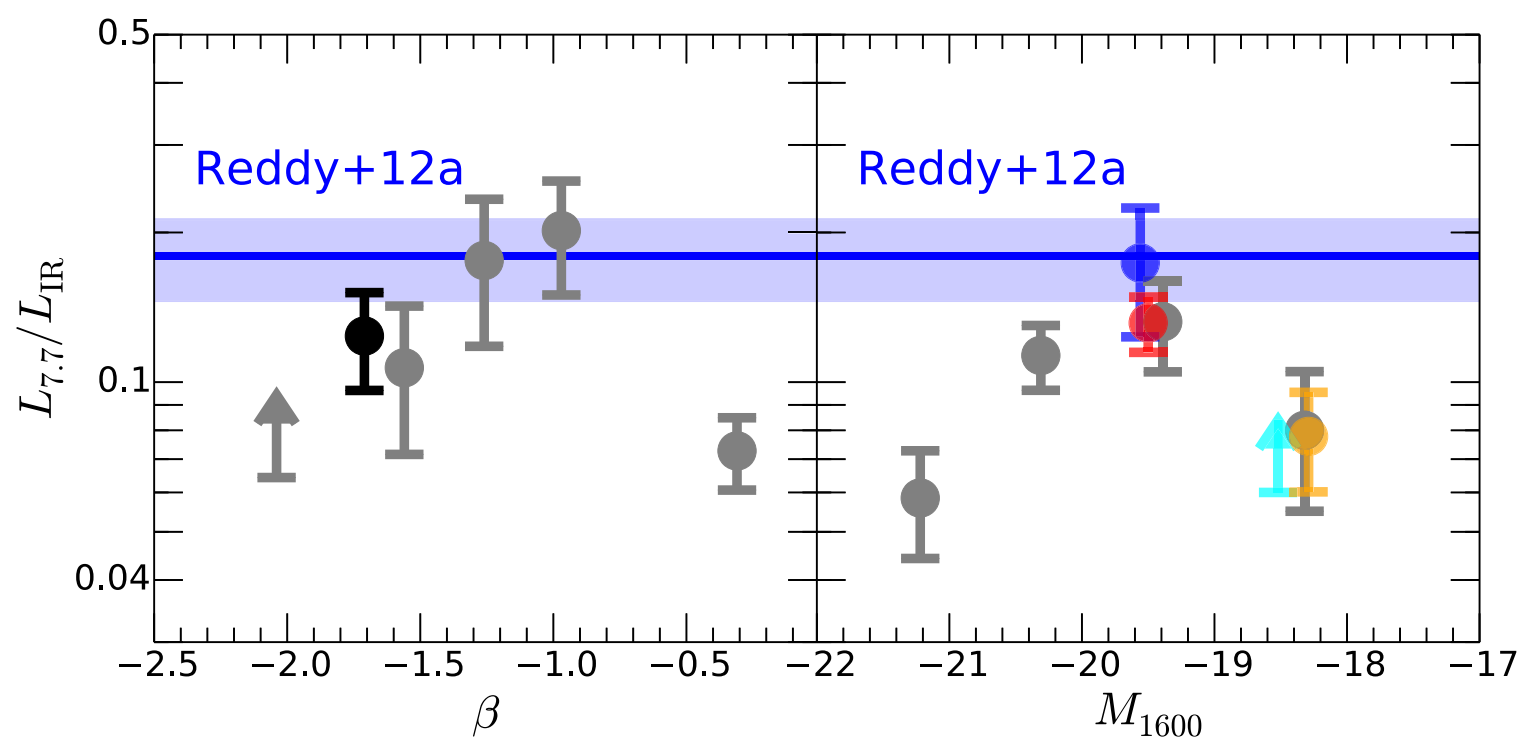

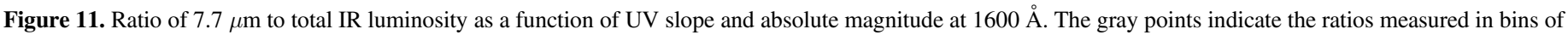

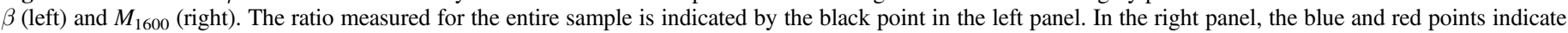

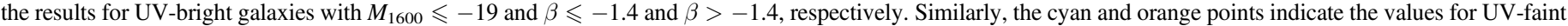

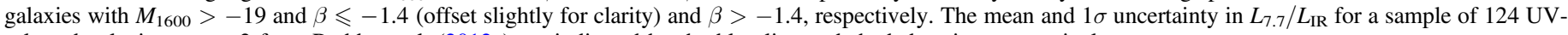
selected galaxies at $z \sim 2$ from Reddy et al. (2012a) are indicated by the blue line and shaded region, respectively.

instrument. A number of early studies of local and $z \sim 1$ galaxies suggested that PAH emission correlates with total dust emission (e.g., Roussel et al. 2001; Förster Schreiber et al. 2003, 2004), though with some variations with metallicity, ionizing intensity, SFR surface density, and stellar population age (e.g., Normand et al. 1995; Helou et al. 2001; Alonso-Herrero et al. 2004; Engelbracht et al. 2005; Hogg et al. 2005; Madden et al. 2006; Draine et al. 2007; Smith et al. 2007; Galliano et al. 2008; Hunt et al. 2010; Sales et al. 2010; Elbaz et al. 2011; Magdis et al. 2013; Seok et al. 2014). Recently, Shivaei et al. (2017) presented the first statistically significant trends showing lower ratios of the $7.7 \mu \mathrm{m}$ to total IR luminosity, $L_{7.7} / L_{\mathrm{IR}}$, with higher ionization intensity, lower gas-phase metallicity, and younger ages for galaxies at $z \sim 2$ from the MOSDEF survey (Kriek et al. 2015). The aforementioned studies have suggested either a delayed enrichment of PAHs in young galaxies or the destruction of PAHs in high-ionization and low-metallicity environments.

In light of these findings, we considered $L_{7.7} / L_{\mathrm{IR}}$ for the predominantly blue, low-luminosity galaxies in our sample. ${ }^{15}$ The galaxies in our sample as a whole exhibit $\left\langle L_{7.7} / L_{\mathrm{IR}}\right\rangle=$ $0.12 \pm 0.03$, similar within $1 \sigma$ to that computed in Reddy et al. (2012a), $\left\langle L_{7.7} / L_{\mathrm{IR}}\right\rangle=0.18 \pm 0.03$, where the latter has been corrected for the difference in this ratio when assuming the

\footnotetext{
15 As virtually all of the galaxies in our sample are undetected in the PACS imaging, we were not able to normalize the $24 \mu \mathrm{m}$ images by $1 / L_{\mathrm{IR}}$ before stacking them (e.g., in the same way that we were able to normalize them by $1 / L_{\mathrm{UV}}$; Section 3$)$. However, as the stacked fluxes are weighted by $1 / L_{\mathrm{UV}}$, and $L_{\mathrm{UV}} \propto \mathrm{SFR} \propto L_{\mathrm{IR}}$ for all but the dustiest galaxies in our sample (Section 5.3), we assumed that the ratio of the average luminosities is similar to the average ratio of the luminosities, i.e., $\left\langle L_{7.7}\right\rangle /\left\langle L_{\mathrm{IR}}\right\rangle \approx\left\langle L_{7.7} / L_{\mathrm{IR}}\right\rangle$ (see discussion in Shivaei et al. 2017). Thus, we simply divided $L_{7.7}$ by $L_{\mathrm{IR}}$ for each stack presented in Table 2 in order to deduce the average ratio $\left\langle L_{7.7} / L_{\mathrm{IR}}\right\rangle$.
}

Elbaz et al. (2011) dust template rather than those of Chary \& Elbaz (2001). However, when divided into bins of UV slope, we find that $\left\langle L_{7.7} / L_{\mathrm{IR}}\right\rangle$ is substantially lower for galaxies with the reddest $\beta$, as well as for the brightest $\left(M_{1600} \leqslant-21\right)$ and faintest $\left(M_{1600}>-19\right)$ galaxies in our sample (Figure 11).

The low ratio $\left(\left\langle L_{7.7} / L_{\mathrm{IR}}\right\rangle=0.07 \pm 0.01\right)$ observed for galaxies with the reddest UV slopes may be related to significant $9.9 \mu \mathrm{m}$ silicate absorption affecting the observed $24 \mu \mathrm{m}$ flux. An alternative explanation invokes an IR luminosity that is boosted in the presence of AGNs, though we consider this possibility unlikely as we have removed obscured AGNs from the sample based on their IRAC colors (Section 2). Regardless, the lower $\left\langle L_{7.7} / L_{\mathrm{IR}}\right\rangle$ found for these red galaxies is partly responsible for a similar low ratio found for the faintest galaxies in our sample, since many of the dustiest galaxies in our sample are also UV-faint (Figure 1). Isolating the UV-faint galaxies with slopes bluer than $\beta=-1.4$ yields an unconstraining lower limit on $\left\langle L_{7.7} / L_{\mathrm{IR}}\right\rangle$.

Finally, we note that the brightest galaxies in our sample $\left(M_{1600} \leqslant-21\right)$ also exhibit a very low $\left\langle L_{7.7} / L_{\mathrm{IR}}\right\rangle=$ $0.06 \pm 0.01$. Such galaxies are on average a factor of 1.6 younger than $M_{1600}>-21$ galaxies $(\approx 500$ versus $\approx 800 \mathrm{Myr}$ ). Thus, their lower mean ratio may be related to a deficit of PAHs due to younger stellar population ages, or may be related to harder ionization fields and/or lower gas-phase metallicities. Unfortunately, we are unable to fully explore how the $L_{7.7}$-to- $L_{\mathrm{IR}}$ ratio varies with age given unconstraining lower limits on this value for galaxies with ages $\lesssim 500$ Myr. Irrespective of the physical causes for changes in the PAH-to-infrared luminosity ratio, our results suggest that caution must be used when adopting a single-valued conversion to recover $L_{\mathrm{IR}}$ from mid-IR measurements. For example, assuming the mean ratio found for our sample would result in $24 \mu \mathrm{m}$ inferred $L_{\mathrm{IR}}$ that are a factor of $\approx 2$ lower than the 
"true" values for the reddest $(\beta>-0.8)$ and UV-brightest $\left(M_{1600} \leqslant-21\right)$ galaxies in our sample.

\section{ORCID iDs}

Naveen A. Reddy (i) https://orcid.org/0000-0001-9687-4973 Pascal A. Oesch (i) https://orcid.org/0000-0001-5851-6649 Rychard J. Bouwens (i) https://orcid.org/0000-00024989-2471

Charles C. Steidel (1) https://orcid.org/0000-0002-4834-7260

Pieter G. van Dokkum (1) https://orcid.org/0000-00028282-9888

Hakim Atek (1) https://orcid.org/0000-0002-7570-0824

Marcella C. Carollo (i) https://orcid.org/0000-0003-1624-7609

Anna Cibinel 1 https://orcid.org/0000-0003-4578-514X

Brad Holden (i) https://orcid.org/0000-0002-6153-3076

Ivo Labbé i https://orcid.org/0000-0002-2057-5376

Erica J. Nelson (1) https://orcid.org/0000-0002-7524-374X

\section{References}

Adelberger, K. L., \& Steidel, C. C. 2000, ApJ, 544, 218

Alavi, A., Siana, B., Richard, J., et al. 2014, ApJ, 780, 143

Alavi, A., Siana, B., Richard, J., et al. 2016, ApJ, 832, 56

Alonso-Herrero, A., Takagi, T., Baker, A. J., et al. 2004, ApJ, 612, 222

Álvarez-Márquez, J., Burgarella, D., Heinis, S., et al. 2016, A\&A, 587, A122

Amanullah, R., Goobar, A., Johansson, J., et al. 2014, ApJL, 788, L21

Andrews, B. H., \& Martini, P. 2013, ApJ, 765, 140

Asplund, M., Grevesse, N., Sauval, A. J., \& Scott, P. 2009, ARA\&A, 47, 481

Baker, A. J., Lutz, D., Genzel, R., Tacconi, L. J., \& Lehnert, M. D. 2001, A\&A, 372, L37

Barger, A. J., Cowie, L. L., \& Richards, E. A. 2000, AJ, 119, 2092

Basu-Zych, A. R., Lehmer, B. D., Hornschemeier, A. E., et al. 2013, ApJ, 774,152

Bertin, E., \& Arnouts, S. 1996, A\&AS, 117, 393

Boquien, M., Buat, V., Boselli, A., et al. 2012, A\&A, 539, A145

Bouwens, R. J., Aravena, M., Decarli, R., et al. 2016a, ApJ, 833, 72

Bouwens, R. J., Illingworth, G. D., Franx, M., et al. 2009, ApJ, 705, 936

Bouwens, R. J., Illingworth, G. D., Franx, M., \& Ford, H. 2007, ApJ, 670, 928

Bouwens, R. J., Illingworth, G. D., Oesch, P. A., et al. 2012, ApJ, 754, 83

Bouwens, R. J., Illingworth, G. D., Oesch, P. A., et al. 2015, ApJ, 811, 140

Bouwens, R. J., Smit, R., Labbé, I., et al. 2016b, ApJ, 831, 176

Brammer, G. B., van Dokkum, P. G., \& Coppi, P. 2008, ApJ, 686, 1503

Brott, I., de Mink, S. E., Cantiello, M., et al. 2011, A\&A, 530, A115

Bruzual, G., \& Charlot, S. 2003, MNRAS, 344, 1000

Buat, V., Giovannoli, E., Heinis, S., et al. 2011, A\&A, 533, A93

Buat, V., Iglesias-Páramo, J., Seibert, M., et al. 2005, ApJL, 619, L51

Buat, V., Marcillac, D., Burgarella, D., et al. 2007, A\&A, 469, 19

Buat, V., Noll, S., Burgarella, D., et al. 2012, A\&A, 545, A141

Buat, V., Takeuchi, T. T., Burgarella, D., Giovannoli, E., \& Murata, K. L. 2009, A\&A, 507, 693

Burgarella, D., Buat, V., \& Iglesias-Páramo, J. 2005, MNRAS, 360, 1413

Burgarella, D., Buat, V., Takeuchi, T. T., Wada, T., \& Pearson, C. 2009, PASJ, 61, 177

Calzetti, D., Armus, L., Bohlin, R. C., et al. 2000, ApJ, 533, 682

Calzetti, D., Kinney, A. L., \& Storchi-Bergmann, T. 1994, ApJ, 429, 582

Capak, P. L., Carilli, C., Jones, G., et al. 2015, Natur, 522, 455

Caputi, K. I., Lagache, G., Yan, L., et al. 2007, ApJ, 660, 97

Carilli, C. L., \& Walter, F. 2013, ARA\&A, 51, 105

Casey, C. M., Narayanan, D., \& Cooray, A. 2014a, PhR, 541, 45

Casey, C. M., Scoville, N. Z., Sanders, D. B., et al. 2014b, ApJ, 796, 95 Chabrier, G. 2003, PASP, 115, 763

Chapman, S. C., Blain, A. W., Smail, I., \& Ivison, R. J. 2005, ApJ, 622, 772 Chapman, S. C., Scott, D., Steidel, C. C., et al. 2000, MNRAS, 319, 318

Chary, R., \& Elbaz, D. 2001, ApJ, 556, 562

Conroy, C., \& Gunn, J. E. 2010, ApJ, 712, 833

Cullen, F., McLure, R. J., Khochfar, S., Dunlop, J. S., \& Dalla Vecchia, C. 2017, MNRAS, 470, 3006

Daddi, E., Dickinson, M., Morrison, G., et al. 2007, ApJ, 670, 156

Dale, D. A., Cohen, S. A., Johnson, L. C., et al. 2009, ApJ, 703, 517

Dale, D. A., \& Helou, G. 2002, ApJ, 576, 159

Davidzon, I., Ilbert, O., Laigle, C., et al. 2017, A\&A, 605, A70
De Barros, S., Reddy, N., \& Shivaei, I. 2016, ApJ, 820, 96 de Barros, S., Schaerer, D., \& Stark, D. P. 2014, A\&A, 563, A81

Dessauges-Zavadsky, M., Zamojski, M., Rujopakarn, W., et al. 2017, A\&A, 605, A81

Domínguez, A., Siana, B., Brooks, A. M., et al. 2015, MNRAS, 451, 839

Donley, J. L., Koekemoer, A. M., Brusa, M., et al. 2012, ApJ, 748, 142

Draine, B. T., Dale, D. A., Bendo, G., et al. 2007, ApJ, 663, 866

Draine, B. T., \& Salpeter, E. E. 1979, ApJ, 231, 438

Dunlop, J. S., McLure, R. J., Biggs, A. D., et al. 2017, MNRAS, 466, 861

Elbaz, D., Dickinson, M., Hwang, H. S., et al. 2011, A\&A, 533, A119

Eldridge, J. J., \& Stanway, E. R. 2009, MNRAS, 400, 1019

Eldridge, J. J., \& Stanway, E. R. 2012, MNRAS, 419, 479

Engelbracht, C. W., Gordon, K. D., Rieke, G. H., et al. 2005, ApJL, 628, L29

Erb, D. K., Shapley, A. E., Pettini, M., et al. 2006, ApJ, 644, 813

Faisst, A. L., Capak, P. L., Yan, L., et al. 2017, ApJ, 847, 21

Faucher-Giguere, C.-A. 2018, MNRAS, 473, 3717

Ferrara, A., Viti, S., \& Ceccarelli, C. 2016, MNRAS, 463, L112

Finkelstein, S. L., Papovich, C., Salmon, B., et al. 2012, ApJ, 756, 164

Forrest, B., Tran, K.-V. H., Tomczak, A. R., et al. 2016, ApJL, 818, L26

Förster Schreiber, N. M., Roussel, H., Sauvage, M., \& Charmandaris, V. 2004, A\&A, 419, 501

Förster Schreiber, N. M., Sauvage, M., Charmandaris, V., et al. 2003, A\&A, 399,833

Fudamoto, Y., Oesch, P. A., Schinnerer, E., et al. 2017, MNRAS, 472, 483

Galliano, F., Dwek, E., \& Chanial, P. 2008, ApJ, 672, 214

Goldader, J. D., Meurer, G., Heckman, T. M., et al. 2002, ApJ, 568, 651

Gordon, K. D., Cartledge, S., \& Clayton, G. C. 2009, ApJ, 705, 1320

Gordon, K. D., Clayton, G. C., Misselt, K. A., Landolt, A. U., \& Wolff, M. J. 2003, ApJ, 594, 279

Grasha, K., Calzetti, D., Andrews, J. E., Lee, J. C., \& Dale, D. A. 2013, ApJ, 773,174

Guo, Y., Rafelski, M., Faber, S. M., et al. 2016, ApJ, 833, 37

Hall, P. B., Anderson, S. F., Strauss, M. A., et al. 2002, ApJS, 141, 267

Hathi, N. P., Ryan, R. E., Jr., Cohen, S. H., et al. 2010, ApJ, 720, 1708

Heinis, S., Buat, V., Béthermin, M., et al. 2013, MNRAS, 429, 1113

Heinis, S., Buat, V., Béthermin, M., et al. 2014, MNRAS, 437, 1268

Helou, G., Malhotra, S., Hollenbach, D. J., Dale, D. A., \& Contursi, A. 2001, ApJL, 548, L73

Henry, A., Scarlata, C., Domínguez, A., et al. 2013, ApJL, 776, L27

Hogg, D. W., Tremonti, C. A., Blanton, M. R., et al. 2005, ApJ, 624, 162

Hopkins, P. F., Kereš, D., Oñorbe, J., et al. 2014, MNRAS, 445, 581

Hunt, L. K., Thuan, T. X., Izotov, Y. I., \& Sauvage, M. 2010, ApJ, 712, 164 Jiang, P., Zhou, H., Ji, T., et al. 2013, AJ, 145, 157

Johnson, B. D., Schiminovich, D., Seibert, M., et al. 2007, ApJS, 173, 392

Jones, A. P. 2004, in ASP Conf. Ser. 309, Astrophysics of Dust, ed. A. N. Witt, G. C. Clayton, \& B. T. Draine (San Francisco, CA: ASP), 347 Kennicutt, R. C., \& Evans, N. J. 2012, ARA\&A, 50, 531

Kewley, L. J., \& Ellison, S. L. 2008, ApJ, 681, 1183

Knudsen, K. K., Richard, J., Kneib, J.-P., et al. 2016, MNRAS, 462, L6

Koekemoer, A. M., Faber, S. M., Ferguson, H. C., et al. 2011, ApJS, 197, 36

Kong, X., Charlot, S., Brinchmann, J., \& Fall, S. M. 2004, MNRAS, 349, 769 Koprowski, M. P., Coppin, K. E. K., Geach, J. E., et al. 2016, ApJL, 828, L21 Kriek, M., \& Conroy, C. 2013, ApJL, 775, L16

Kriek, M., Shapley, A. E., Reddy, N. A., et al. 2015, ApJS, 218, 15

Kriek, M., van Dokkum, P. G., Labbé, I., et al. 2009, ApJ, 700, 221

Kurczynski, P., Gawiser, E., Rafelski, M., et al. 2014, ApJL, 793, L5

Lee, J. C., Ly, C., Spitler, L., et al. 2012, PASP, 124, 782

Leitherer, C., Ekström, S., Meynet, G., et al. 2014, ApJS, 212, 14

Levesque, E. M., Leitherer, C., Ekstrom, S., Meynet, G., \& Schaerer, D. 2012, ApJ, 751, 67

Madden, S. C., Galliano, F., Jones, A. P., \& Sauvage, M. 2006, A\&A, 446, 877

Magdis, G. E., Elbaz, D., Daddi, E., et al. 2010, ApJ, 714, 1740

Magdis, G. E., Rigopoulou, D., Helou, G., et al. 2013, A\&A, 558, A136

Magnelli, B., Popesso, P., Berta, S., et al. 2013, A\&A, 553, A132

Maiolino, R., Carniani, S., Fontana, A., et al. 2015, MNRAS, 452, 54

Maiolino, R., Nagao, T., Grazian, A., et al. 2008, A\&A, 488, 463

Maseda, M. V., van der Wel, A., Rix, H.-W., et al. 2014, ApJ, 791, 17

McKee, C. 1989, in IAU Symp. 135, Interstellar Dust, ed. L. J. Allamandola \& A. G. G. M. Tielens, 431

McLure, R. J., Dunlop, J. S., Cullen, F., et al. 2017, MNRAS, submitted (arXiv:1709.06102)

Meurer, G. R., Heckman, T. M., \& Calzetti, D. 1999, ApJ, 521, 64

Momcheva, I. G., Brammer, G. B., van Dokkum, P. G., et al. 2016, ApJS, 225, 27

Muñoz-Mateos, J. C., Gil de Paz, A., Boissier, S., et al. 2009, ApJ, 701, 1965 
Nakajima, K., Ellis, R. S., Iwata, I., et al. 2016, ApJL, 831, L9 Nandra, K., Mushotzky, R. F., Arnaud, K., et al. 2002, ApJ, 576, 625

Nelson, E. J., van Dokkum, P. G., Momcheva, I. G., et al. 2016, ApJL, 817, L9

Noeske, K. G., Weiner, B. J., Faber, S. M., et al. 2007, ApJL, 660, L43

Noll, S., Pierini, D., Cimatti, A., et al. 2009, A\&A, 499, 69

Normand, P., Rouan, D., Lacombe, F., \& Tiphene, D. 1995, A\&A, 297, 311

Oesch, P. A., Bouwens, R. J., Illingworth, G. D., et al. 2010, ApJL, 709, L16 Oke, J. B., \& Gunn, J. E. 1983, ApJ, 266, 713

Overzier, R. A., Heckman, T. M., Wang, J., et al. 2011, ApJL, 726, L7

Pannella, M., Carilli, C. L., Daddi, E., et al. 2009, ApJL, 698, L116

Pannella, M., Elbaz, D., Daddi, E., et al. 2015, ApJ, 807, 141

Penner, K., Dickinson, M., Pope, A., et al. 2012, ApJ, 759, 28

Pentericci, L., Carniani, S., Castellano, M., et al. 2016, ApJL, 829, L11

Pettini, M., Kellogg, M., Steidel, C. C., et al. 1998, ApJ, 508, 539

Pettini, M., Steidel, C. C., Adelberger, K. L., Dickinson, M., \& Giavalisco, M. 2000, ApJ, 528, 96

Price, S. H., Kriek, M., Brammer, G. B., et al. 2014, ApJ, 788, 86

Rafelski, M., Teplitz, H. I., Gardner, J. P., et al. 2015, AJ, 150, 31

Reddy, N., Dickinson, M., Elbaz, D., et al. 2012a, ApJ, 744, 154

Reddy, N. A., Erb, D. K., Pettini, M., Steidel, C. C., \& Shapley, A. E. 2010, ApJ, 712, 1070

Reddy, N. A., Erb, D. K., Steidel, C. C., et al. 2005, ApJ, 633, 748

Reddy, N. A., Kriek, M., Shapley, A. E., et al. 2015, ApJ, 806, 259

Reddy, N. A., Pettini, M., Steidel, C. C., et al. 2012b, ApJ, 754, 25

Reddy, N. A., \& Steidel, C. C. 2004, ApJL, 603, L13

Reddy, N. A., \& Steidel, C. C. 2009, ApJ, 692, 778

Reddy, N. A., Steidel, C. C., Erb, D. K., Shapley, A. E., \& Pettini, M. 2006a, ApJ, 653, 1004

Reddy, N. A., Steidel, C. C., Fadda, D., et al. 2006b, ApJ, 644, 792

Reddy, N. A., Steidel, C. C., Pettini, M., et al. 2008, ApJS, 175, 48

Reddy, N. A., Steidel, C. C., Pettini, M., \& Bogosavljević, M. 2016a, ApJ, 828, 107

Reddy, N. A., Steidel, C. C., Pettini, M., Bogosavljević, M., \& Shapley, A. E. 2016b, ApJ, 828, 108

Rieke, G. H., Alonso-Herrero, A., Weiner, B. J., et al. 2009, ApJ, 692, 556 Robertson, B. E., Furlanetto, S. R., Schneider, E., et al. 2013, ApJ, 768, 71

Roussel, H., Sauvage, M., Vigroux, L., \& Bosma, A. 2001, A\&A, 372, 427

Sales, D. A., Pastoriza, M. G., \& Riffel, R. 2010, ApJ, 725, 605

Salmon, B., Papovich, C., Long, J., et al. 2016, ApJ, 827, 20

Sanders, R. L., Shapley, A. E., Kriek, M., et al. 2015, ApJ, 799, 138

Schaerer, D., Boone, F., Zamojski, M., et al. 2015, A\&A, 574, A19

Schaerer, D., de Barros, S., \& Sklias, P. 2013, A\&A, 549, A4

Schechter, P. 1976, ApJ, 203, 297

Schreiber, C., Pannella, M., Elbaz, D., et al. 2015, A\&A, 575, A74
Seibert, M., Heckman, T. M., \& Meurer, G. R. 2002, AJ, 124, 46 Seibert, M., Martin, D. C., Heckman, T. M., et al. 2005, ApJL, 619, L55

Seok, J. Y., Hirashita, H., \& Asano, R. S. 2014, MNRAS, 439, 2186

Shao, L., Lutz, D., Nordon, R., et al. 2010, A\&A, 518, L26

Shipley, H. V., Papovich, C., Rieke, G. H., Brown, M. J. I., \& Moustakas, J. 2016, ApJ, 818, 60

Shivaei, I., Reddy, N., Shapley, A., et al. 2017, ApJ, 837, 157

Shivaei, I., Reddy, N. A., Shapley, A. E., et al. 2015, ApJ, 815, 98

Siana, B., Smail, I., Swinbank, A. M., et al. 2009, ApJ, 698, 1273

Siana, B., Teplitz, H. I., Chary, R.-R., Colbert, J., \& Frayer, D. T. 2008, ApJ, 689, 59

Skelton, R. E., Whitaker, K. E., Momcheva, I. G., et al. 2014, ApJS, 214, 24

Sklias, P., Zamojski, M., Schaerer, D., et al. 2014, A\&A, 561, A149

Smit, R., Bouwens, R. J., Franx, M., et al. 2012, ApJ, 756, 14

Smith, J. D. T., Draine, B. T., Dale, D. A., et al. 2007, ApJ, 656, 770

Sofia, U. J., Wolff, M. J., Rachford, B., et al. 2005, ApJ, 625, 167

Sparre, M., Hayward, C. C., Feldmann, R., et al. 2017, MNRAS, 466, 88

Stanway, E. R., Eldridge, J. J., \& Becker, G. D. 2016, MNRAS, 456, 485

Stark, D. P., Walth, G., Charlot, S., et al. 2015, MNRAS, 454, 1393

Steidel, C. C., Adelberger, K. L., Giavalisco, M., Dickinson, M., \& Pettini, M. 1999, ApJ, 519, 1

Steidel, C. C., Rudie, G. C., Strom, A. L., et al. 2014, ApJ, 795, 165

Steidel, C. C., Strom, A. L., Pettini, M., et al. 2016, ApJ, 826, 159

Teplitz, H. I., Rafelski, M., Kurczynski, P., et al. 2013, AJ, 146, 159

Todini, P., \& Ferrara, A. 2001, MNRAS, 325, 726

Tremonti, C. A., Heckman, T. M., Kauffmann, G., et al. 2004, ApJ, 613 898

van Dokkum, P., Brammer, G., Momcheva, I., et al. 2013, arXiv:1305.2140

Wang, B., \& Heckman, T. M. 1996, ApJ, 457, 645

Watson, D., Christensen, L., Knudsen, K. K., et al. 2015, Natur, 519, 327

Weisz, D. R., Johnson, B. D., Johnson, L. C., et al. 2012, ApJ, 744, 44

Whitaker, K. E., Franx, M., Leja, J., et al. 2014, ApJ, 795, 104

Wilkins, S. M., Bunker, A., Coulton, W., et al. 2013, MNRAS, 430 2885

Wilkins, S. M., Bunker, A. J., Stanway, E., Lorenzoni, S., \& Caruana, J. 2011, MNRAS, 417, 717

Williams, R. J., Quadri, R. F., Franx, M., van Dokkum, P., \& Labbé, I. 2009, ApJ, 691, 1879

Willott, C. J., Carilli, C. L., Wagg, J., \& Wang, R. 2015, ApJ, 807, 180

Windhorst, R. A., Cohen, S. H., Hathi, N. P., et al. 2011, ApJS, 193, 27

Wuyts, S., Förster Schreiber, N. M., Lutz, D., et al. 2011, ApJ, 738, 106

Xue, Y. Q., Luo, B., Brandt, W. N., et al. 2011, ApJS, 195, 10

Zafar, T., Møller, P., Watson, D., et al. 2015, A\&A, 584, A100

Zeimann, G. R., Ciardullo, R., Gronwall, C., et al. 2015, ApJ, 814, 162 